\title{
Graffiti mochicas en la Huaca Cao Viejo, Complejo El Brujo
}

Graffiti mochicas de la Huaca Cao Viejo, Complexe Archéologique Du Brujo Mochica Graffiti in Huaca Cao Viejo, El Brujo Complex

Régulo Franco Jordán, César Gálvez Mora y Segundo Vásquez Sánchez

\section{OpenEdition}

\section{Journals}

Edición electrónica

URL: http://journals.openedition.org/bifea/7155

DOI: $10.4000 /$ bifea. 7155

ISSN: 2076-5827

Editor

Institut Français d'Études Andines

Edición impresa

Fecha de publicación: 1 agosto 2001

Paginación: 359-395

ISSN: 0303-7495

\section{Referencia electrónica}

Régulo Franco Jordán, César Gálvez Mora y Segundo Vásquez Sánchez, "Graffiti mochicas en la Huaca Cao Viejo, Complejo El Brujo », Bulletin de l'Institut français d'études andines [En línea], 30 (2) | 2001, Publicado el 08 agosto 2001, consultado el 08 diciembre 2020. URL : http:// journals.openedition.org/bifea/7155; DOI : https://doi.org/10.4000/bifea.7155

\section{(c) $(1)$}

Les contenus du Bulletin de l'Institut français d'études andines sont mis à disposition selon les termes de la licence Creative Commons Attribution - Pas d'Utilisation Commerciale - Pas de Modification 4.0 International. 


\title{
GRAFFITI MOCHICAS EN LA HUACA CAO VIEJO, COMPLEJO EL BRUJO
}

\author{
Régulo FRANCO JORDÁN*, César GÁLVEZ MORA**, \\ Segundo VÁSQUEZ SÁNCHEZ****
}

\section{Resumen}

En las superficies de muros y columnas de cuatro de los siete edificios superpuestos (E, D, B, A) que forman la Huaca Cao Viejo, se han identificado graffiti en una amplia variedad de diseños. Se discute el propósito de su elaboración, la técnica, asociaciones, cronología relativa, y su recurrencia en otros sitios monumentales de la costa peruana.

Palabras claves: Graffiti, diseños incisos, Mochica, El Brujo, relieves polícromos, pintura mural.

\section{GRAFFITI MOCHICAS DE LA HUACA CAO VIEJO, COMPLEXE ARCHÉOLOGIQUE DU BRUJO}

\section{Résumé}

Sur des murs et colonnes de quatre des sept édifices superposés (E, D, B, A) qui forment la huaca Cao Viejo, nous avons identifié des graffiti qui représentent une grande variété de dessins. Nous analysons actuellement leur élaboration, les techniques employées, leur mise en relation, leur chronologie relative et leur présence dans d'autres sites monumentaux de la côte péruvienne.

Mots clés : Graffiti, dessins incisés, Mochica, El Brujo, reliefs polychromes, pinture murale.

\section{MOCHICA GRAFFITI IN HUACA CAO VIEJO, EL BRUJO COMPLEX}

\begin{abstract}
On the surfaces and columns of four among seven superimposed phases (E, D, B, A) that form the Huaca Cao Viejo, graffiti representing a great variety of designs were discovered. We discuss their relevance, their significance, the techniques used in creating them, their relative chronology, and their use in other monumental buildings on the northern Peruvian coast.
\end{abstract}

Key Words: Graffiti, incised designs, Mochica, El Brujo, polychrome friezes, murals.

*Fundación A. N. Wiese: Canaval y Moreyra 522, Piso 16, Torre Wiese, Lima 27, Perú. E-mail: rfrancj@hotmail.com

${ }^{* *}$ Instituto Nacional de Cultura/La Libertad: Jr. Independencia 572, Trujillo, Perú. E-mail: cegalmor@latinmail.com

*** Universidad Nacional de Trujillo: Juan Pablo II s/n, Urb. San Andrés, Trujillo, Perú. E-mail: svasqs@usa.net 


\section{INTRODUCCIÓN}

En la costa norte del Perú, el complejo arqueológico El Brujo es uno de los sitios claves para entender el desarrollo de una secuencia cultural que sobrepasa los 4500 años de antigüedad (Fig. 1). Las investigaciones realizadas de 1990 al presente (Franco et al., 1994a; 1996; 1998a; 1998b; 1998c; 1998d; 1999a; 1999b; Franco, 1998a; Franco \& Murga, 1998) vienen aportando pruebas sobre la ocupación mochica de este sitio, probablemente el de mayor relevancia en el valle de Chicama, donde destacan por su monumentalidad las huacas El Brujo (huaca Cortada) y Cao Viejo (huaca Blanca).

La huaca Cao Viejo fue la primera que brindó información consistente de la existencia de relieves polícromos en un edificio mochica de la costa norte, que

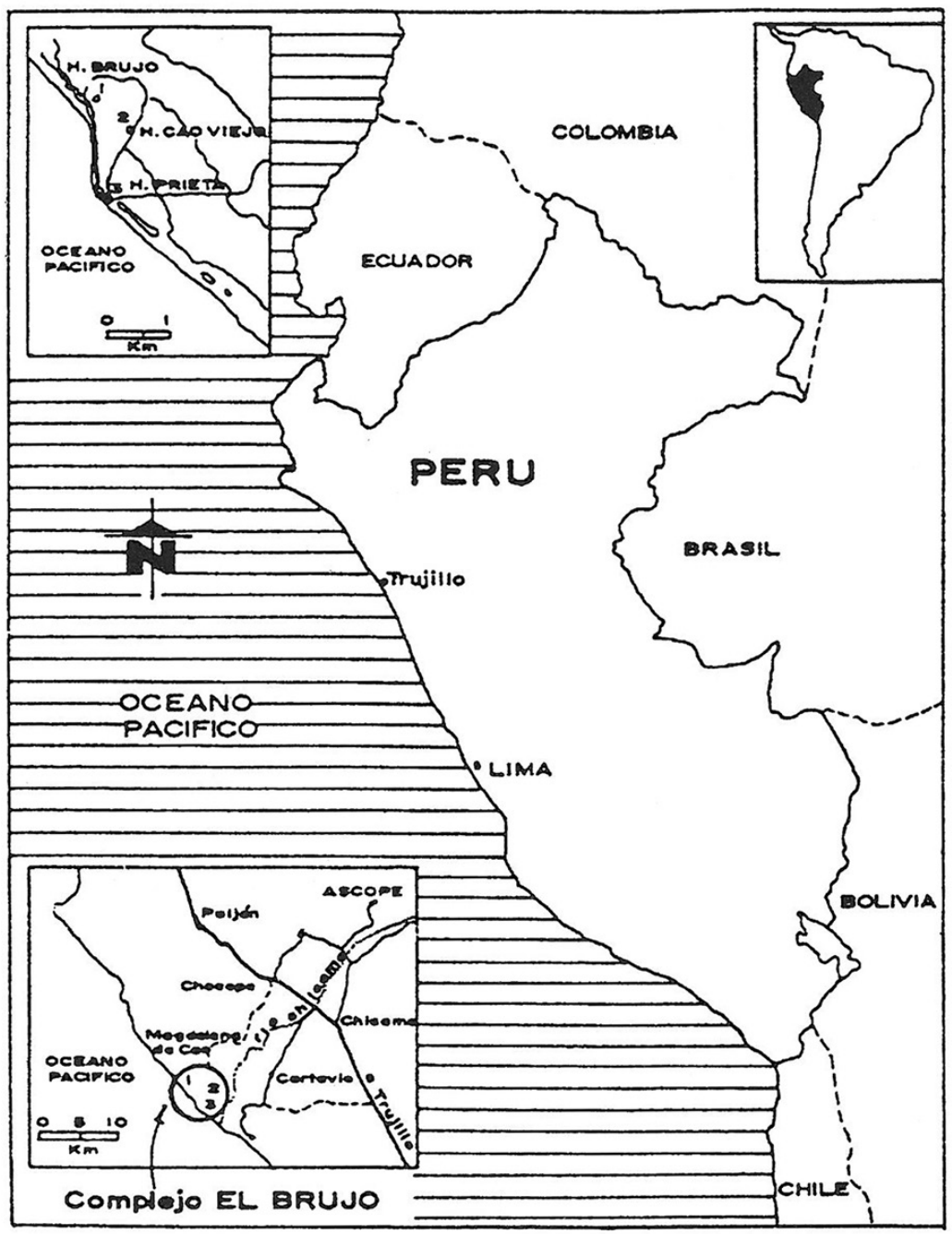

Fig. 1 - Ubicación del complejo arqueológico El Brujo, valle de Chicama. 
representan las expresiones murales más conspicuas del mundo religioso mochica en el valle de Chicama.

En la huaca Cao Viejo (Fig. 2) se ha identificado una secuencia de siete edificios superpuestos (A-G), construidos aproximadamente entre el siglo I al VIII d. C., dos de los cuales se conocen con más detalle (A y D). El modelo arquitectónico básico consiste en una pirámide trunca escalonada, una plaza ceremonial y su anexo este (Fig. 3). En los últimos años, las excavaciones realizadas en la Plataforma Superior (cima) de la pirámide han ampliado la información sobre este modelo y la decoración de sus espacios. Aquí también se han hallado entierros humanos y objetos de uso ceremonial (Franco et al., 1997; 1998a; 1999b).

En este artículo se informa en detalle de la presencia de un número importante de diseños grabados, conocidos como graffiti (Franco et al., 1994b), asociados a los relieves polícromos y superficies monócromas, en cuatro de los siete edificios superpuestos (A, B, D, E). Asimismo se reporta un graffiti en el paramento norte del Montículo 2 (Arévalo et al., 1998: 48-49), un edificio menor localizado a $300 \mathrm{~m}$. al norte de la Huaca Cao Viejo, el mismo que es coetáneo con el Edificio A, el más tardío en la secuencia constructiva de esta huaca. El diseño corresponde a un personaje (Fig. 4A, 5) que ha sido identificado como un "mensajero" (Larco, 1938), "corredores" (Hocquenghem, 1987: 100-101) o "corredor ritual" (Donnan \& McClelland, 1999: fig. 4.99).

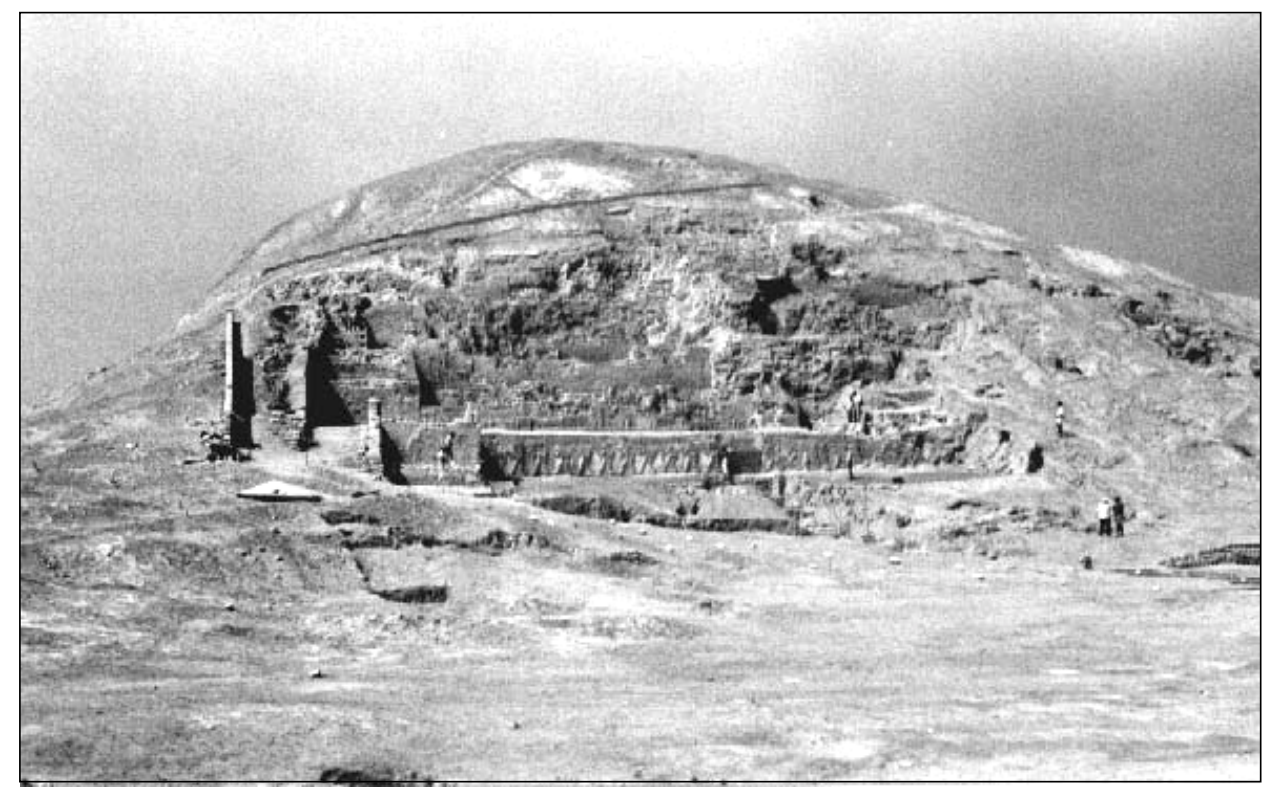

Fig. 2 - Vista del frontis norte de la huaca Cao Viejo, sin cubiertas (1993). 


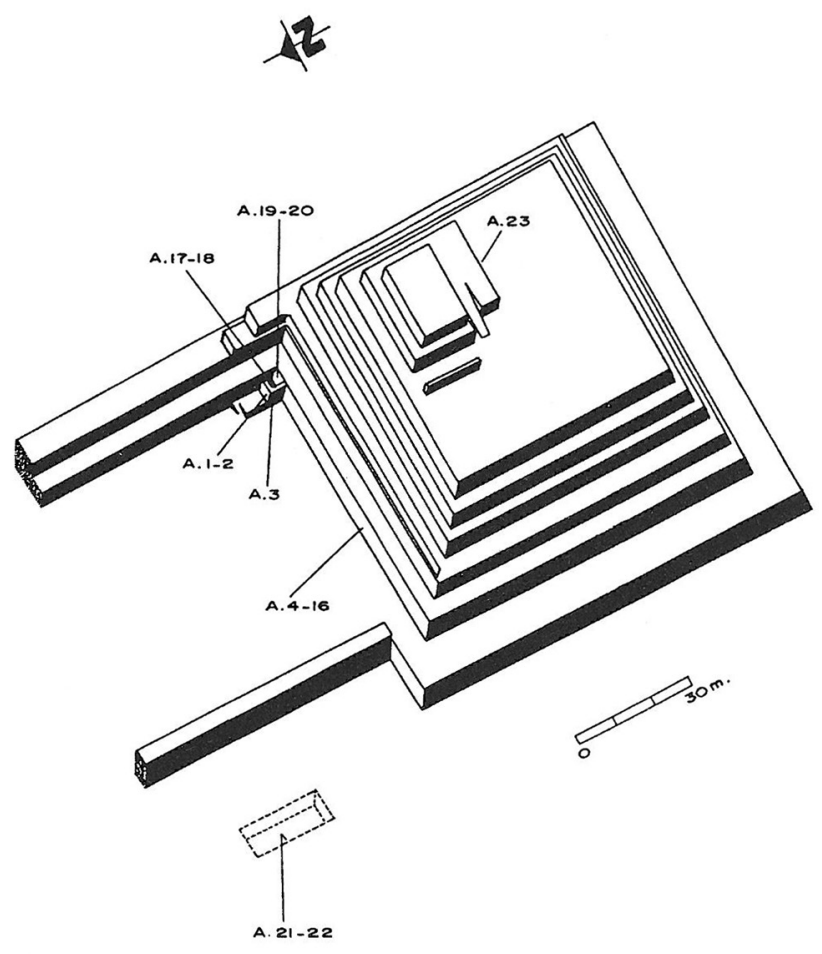

Fig. 3 - Ubicación de graffiti en un dibujo isométrico reconstructivo del Edificio A, huaca Cao Viejo.

En este texto se da a conocer los graffiti mejor definidos y próximos entre sí. Debemos mencionar que un buen número de ellos se hallan dispersos o en mal estado de conservación, lo cual ha dificultado el registro gráfico, y por eso no forman parte de este reporte.

\section{ANTECEDENTES}

Dos de las menciones más antiguas de diseños incisos en la costa norte, proceden del sitio de Gallinazo (V-59), en el valle de Virú. Bennett (1950: fig. 4) presenta motivos de serpientes estilizadas (algunas bicéfalas) y símbolos escalonados; y UbbelohdeDöering (1957: 410) publica motivos indeterminados procedentes del mismo sitio.

Para el valle de Jequetepeque, este último autor (Ubbelohde-Döering, 1957: fig. 1-3) describe tres conjuntos de motivos en mal estado de conservación, en la huaca 31 del complejo Pacatnamú: elementos antropomorfos y líneas, símbolos escalonados y líneas diversas, así como un motivo zoomorfo (ver también Hecker \& Hecker, 1995). De otro lado, en un reciente monitoreo del estado de conservación de la huaca Dos Cabezas, se ha localizado un graffiti en un paramento mal conservado y pintado de 


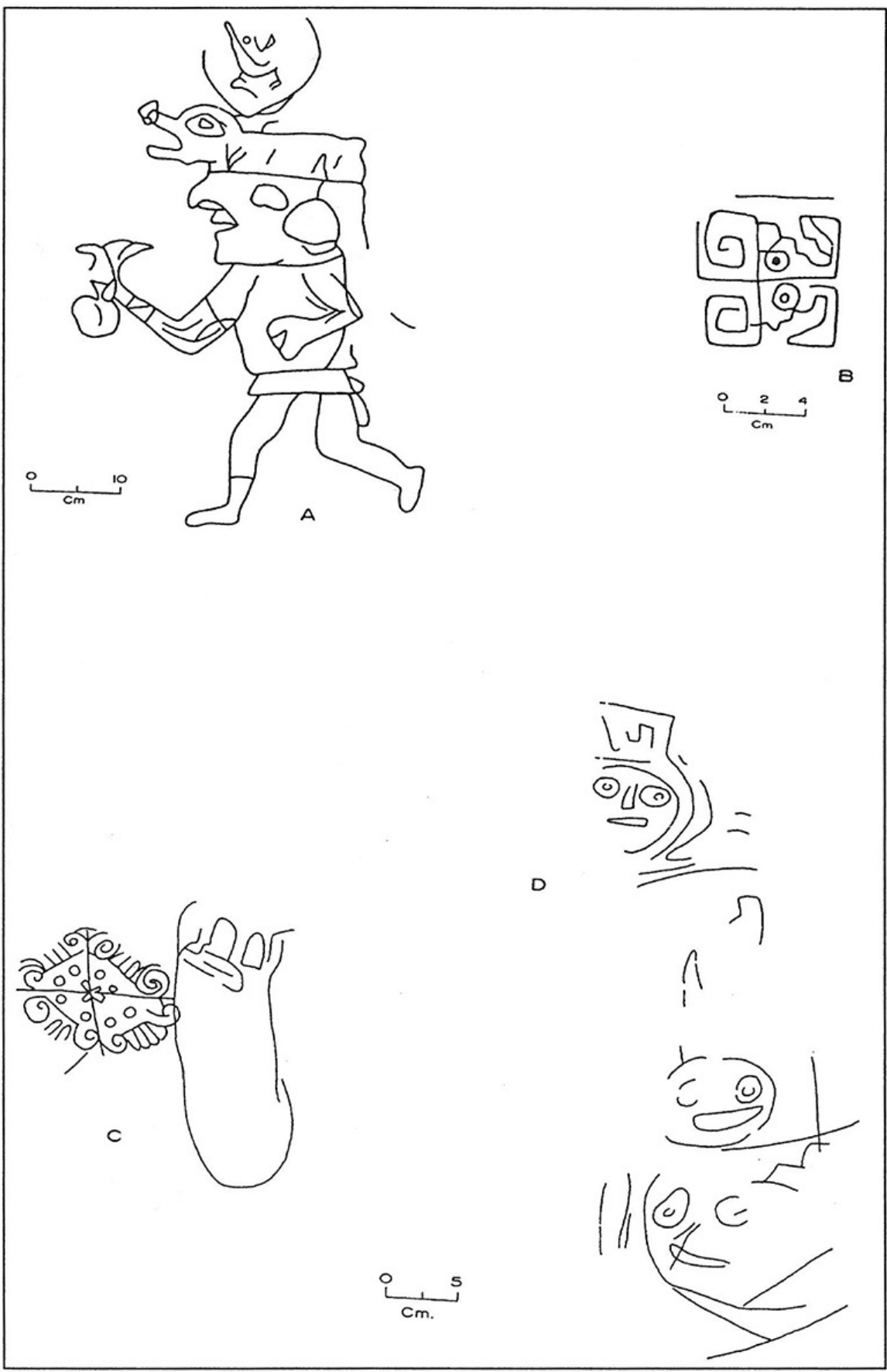

Fig. 4 - Graffiti del Montículo 2 (A); frontis norte del Edificio E: E1 (B); Plataforma Superior: columnas pintadas del patio ceremonial: E2 (C), E3 (D). 


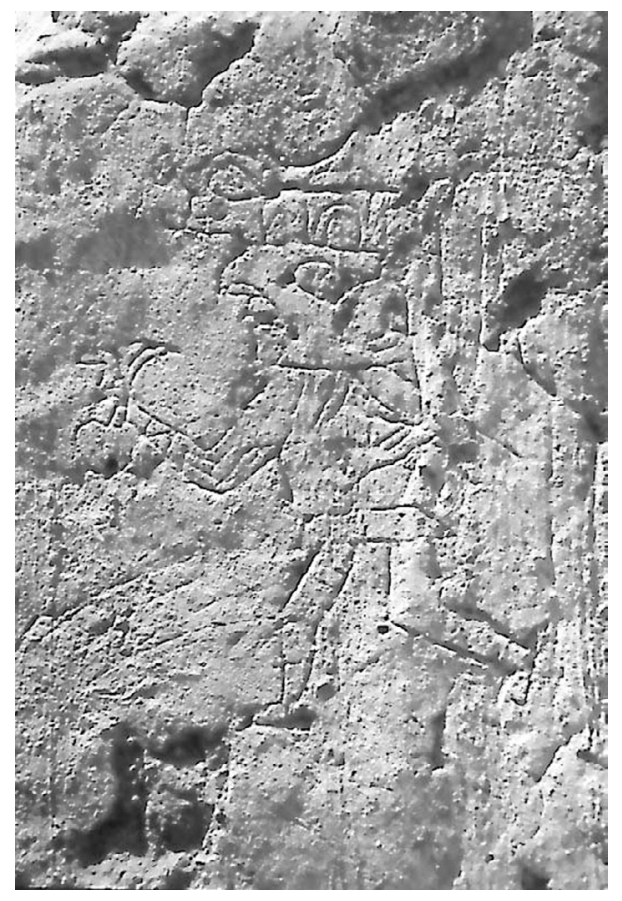

\section{Fig. 5 - Corredor elaborado en el paramento norte del Montículo 2, complejo El Brujo (ver Fig. 4A).}

blanco, el cual se ubica en la cima del edificio y al interior del forado colonial (Ronald Salas, comunicación personal, febrero 2001). En el mismo sitio, Donnan \& Cock (1997: 21, fig. 10) registraron graffiti en el enlucido de barro de las paredes norte y este de la plataforma elevada de un pequeño montículo de ocupación Lambayeque, ubicado en el Sector R del Complejo. Las representaciones corresponden a una figura antropomorfa de perfil, y a un tocado en forma de media luna, así como otras líneas poco definidas.

En Lambayeque, Donnan (1972: 88-91) describió este tipo de diseños en los murales polícromos de la huaca Facho o Mayanga de Batán Grande, en el fondo de los nichos, en las paredes laterales, así como en la fachada y en algunos muros, resultando difícil en este caso definir la relación cronológica de las incisiones en cuanto al pintado de las paredes. Se trata de diseños escalonados y escaleriformes los que podrían corresponder a la tradición mochica (vide Bonavia, 1974: fig. 52). De igual manera, Donnan (1989: 127) publica diseños incisos en el muro norte y en la esquina noroeste del patio de la huaca Chornancap, los que se hicieron sobre las superficies pintadas de blanco, y representan aves, una figura antropomorfa y elementos geométricos. Por su parte, Shimada (1989: 177-180, fig. 8, 10 a, b) presenta los diseños incisos de la huaca Soledad de Batán Grande, de un patrón básico geométrico que el autor relaciona con el sistema de ceques y uno de los geoglifos Nazca, aunque no proporciona mayores argumentos en favor de ello. En este caso, nos parece difícil establecer una comparación entre estos graffiti y el complejo sistema de ceques Inca. 
En el paramento sur de la Plataforma I de la huaca de la Luna, valle de Moche, existe un graffiti que representa un ave marina cogiendo un pez, a la izquierda del ícono principal contenido en un rombo (ver Uceda \& Mujica, 1994: carátula del libro). Este y otros diseños inéditos de la huaca de La Luna aún no han sido descritos ni explicados en su contexto arquitectónico, siendo Campana (1994: fig. 151), el primero que llamó la atención sobre el particular.

Finalmente, un registro fotográfico presenta graffiti en uno de los paramentos decorados de la huaca Pañamarca, en el valle de Nepeña, detrás y encima del lomo de la figura denominada "caracol felínico" por Bonavia (1974: 55) (vide Schaedel, 1970: 115).

Graffiti también existen en la costa central, en edificios ceremoniales más tardíos que la huaca Cao Viejo. En la Pirámide con Rampa No 2 de Pachacamac, aparecen sobre un muro lateral al volumen aterrazado. Se trata de un conjunto de motivos reducidos, especialmente líneas y trazos simples, quizás como representaciones de figuras antropomorfas y zoomorfas (Franco, 1998b: fig. 49a-c).

\section{TÉCNICA DE ELABORACIÓN}

Los diseños grabados aparecen en dos formas:

1. En revoque fresco (o humedecido), que permite trazos firmes con el uso de un instrumento con un extremo fino y redondeado. Es probable que algunos de estos diseños sean coetáneos con el uso y función original de los paramentos, en especial los graffiti del Recinto 1 (Edificio A) ubicados en la franja roja vertical que delimita el borde oeste del Tema Complejo 1 (Fig. 6A y 6B, 7). Pero los diseños que están en las piernas de uno de los guerreros (Fig. 8F, 8G), si bien han sido grabados como parte de la decoración de este personaje, no corresponden a un graffiti propiamente dicho, aunque ha sido incluido en este artículo con fines comparativos.

2. En revoque seco y pintado, mediante el uso de un instrumento cortante para rayar la superficie. En este caso se trata de graffiti realizados de manera espontánea, que a veces copian las representaciones de los relieves. Estos graffiti fueron elaborados en un momento posterior a la culminación de los relieves polícromos (edificios A, D) y después de la aplicación de pintura monócroma y polícroma en los paramentos (edificios A, B, D, E), ya que las incisiones no están cubiertas por pintura. Es probable que los graffiti del edificio B —un proyecto arquitectónico inconcluso-, hayan sido elaborados al detenerse el proceso constructivo, y posteriormente fueron cubiertos con el relleno de adobes tramados del edificio A. En cuanto al edificio A, es muy probable que algunos graffiti fueran realizados durante su funcionamiento y también en los primeros momentos del abandono.

\section{DESCRIPCIÓN DE LOS DISEÑOS}

Hasta la actualidad, se han identificado 3 graffiti en los paramentos del edificio E, 10 en el D (Fig. 9), 7 en el B (Fig. 10) y 23 en el A (Fig. 3). A continuación se presenta cada uno de ellos con sus respectivos datos sobre: 


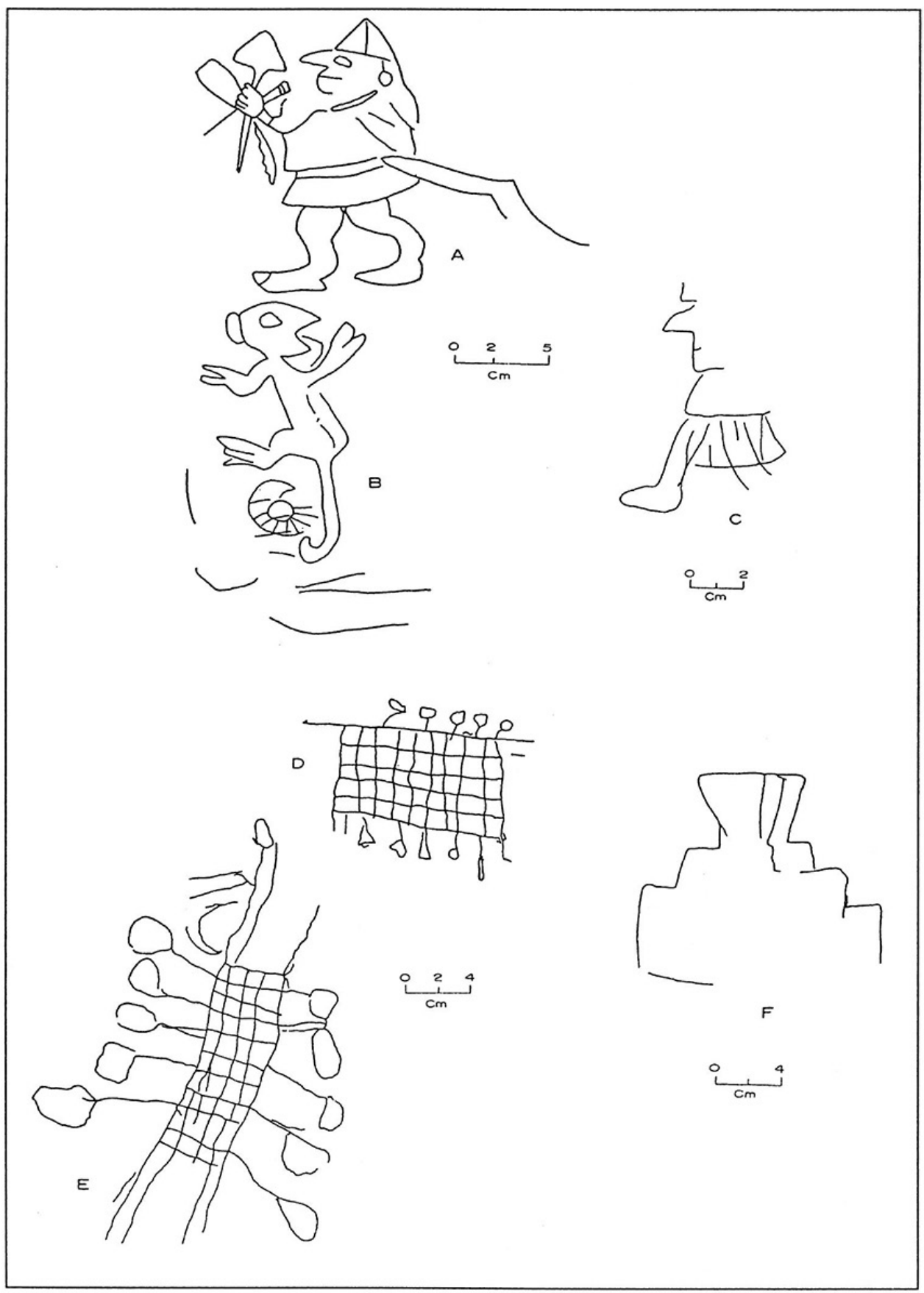

Fig. 6 - Graffiti del Recinto 1 (Plaza Ceremonial) del edificio A: A1 (A), A2 (B), A3 (C), A4 (D); frontis norte: procesión de prisioneros y guerreros: A5 (E), A6 (F). 


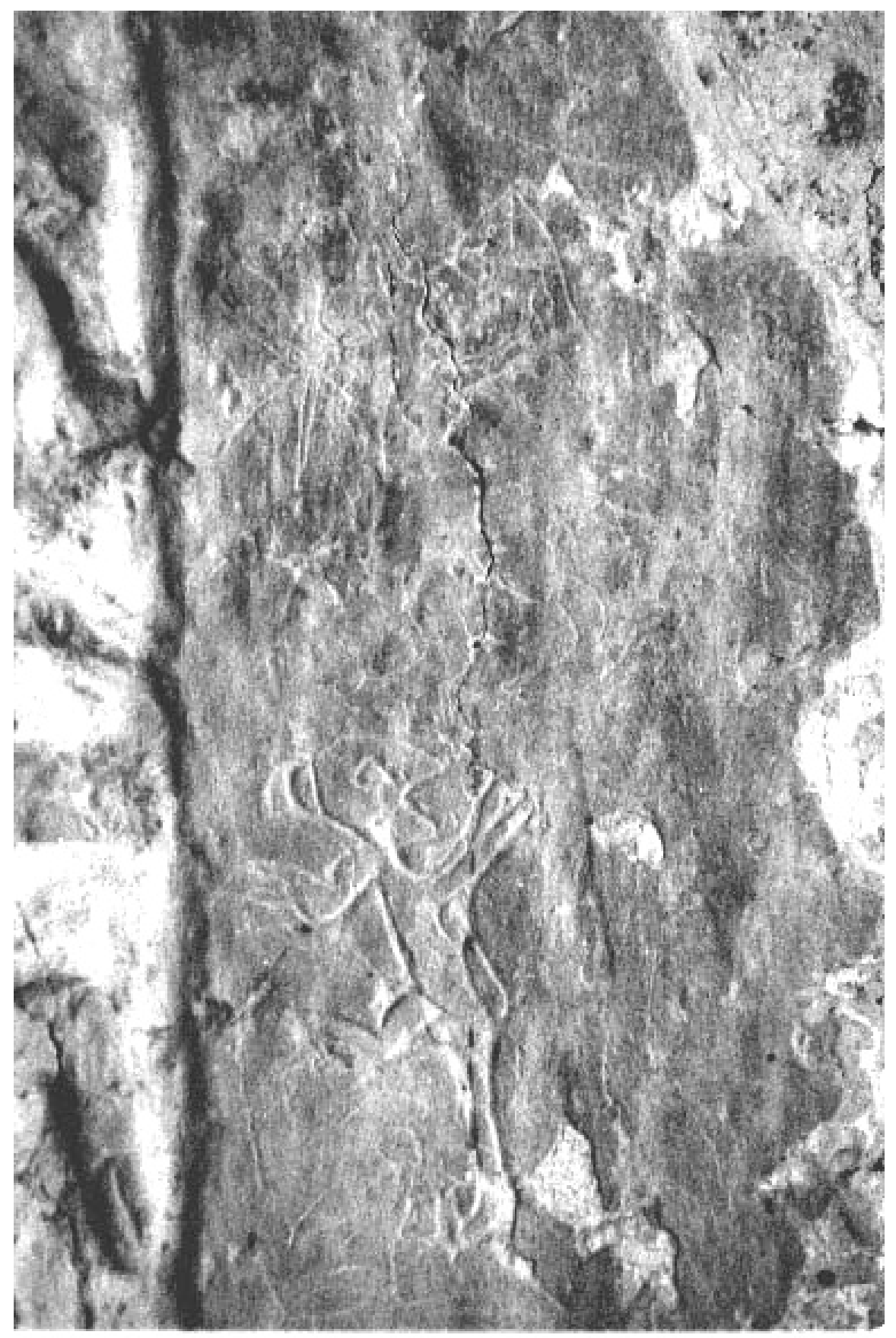

Fig. 7 - Guerrero (Diseño A1, Fig. 11) y motivo zoomorfo (Diseño A2, Fig. 11B) copiado del Tema Complejo 1. 


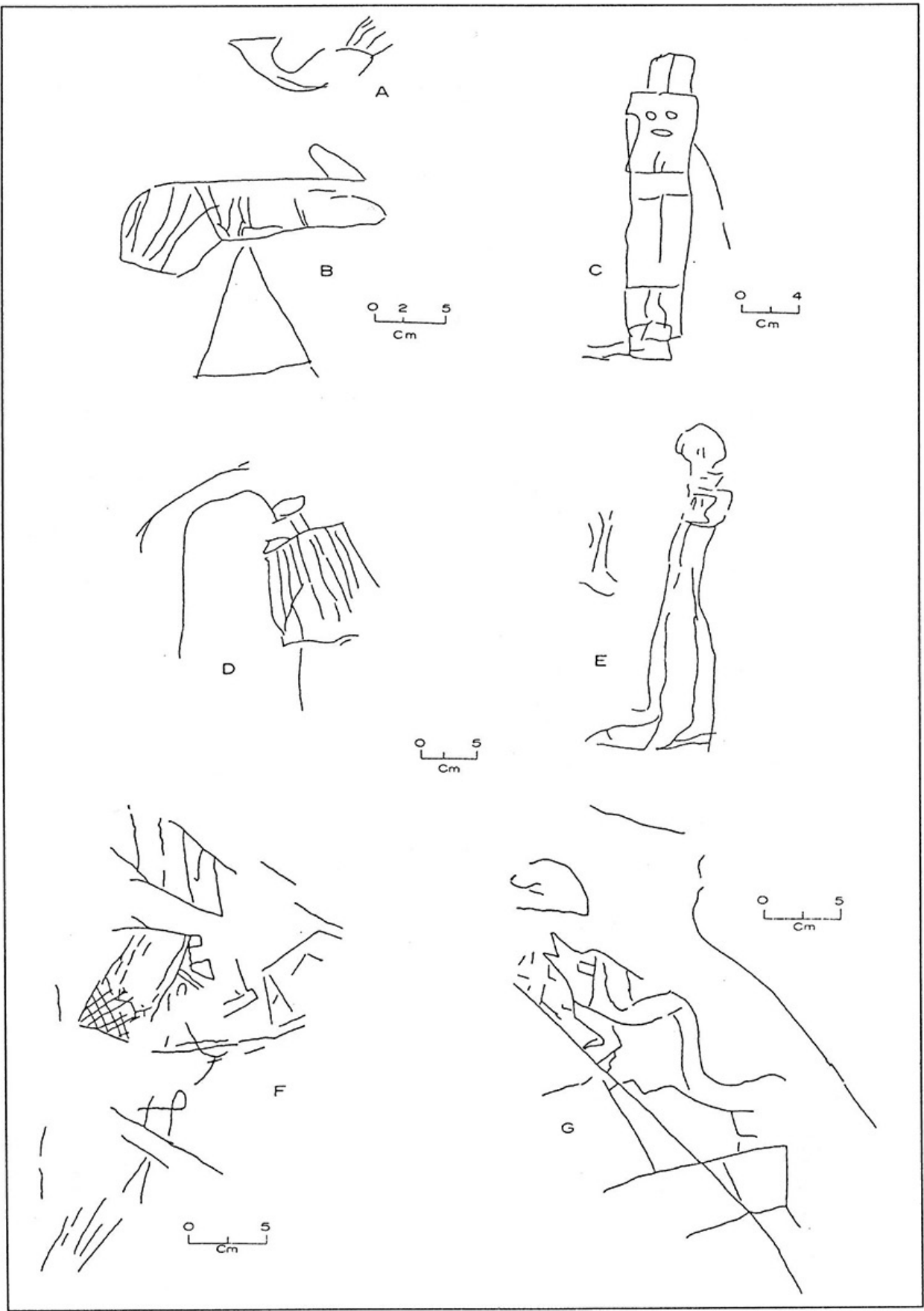

Fig. 8 - Graffiti del frontis norte del edificio A: procesión de prisioneros y guerreros: A7 (A), A8 (B), A9 (C), A10 (D), A11 (E), A12 (F), A13 (G). 


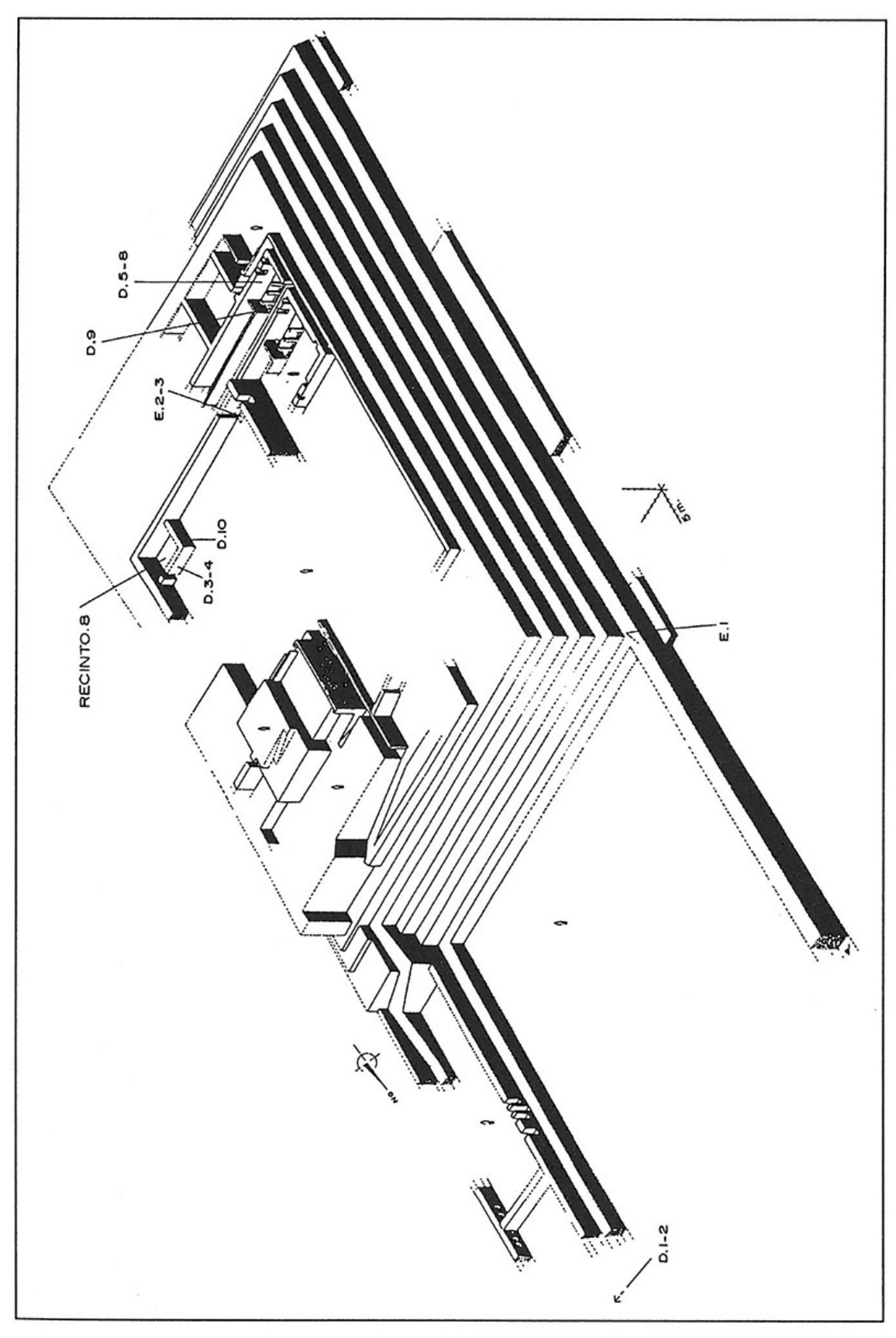

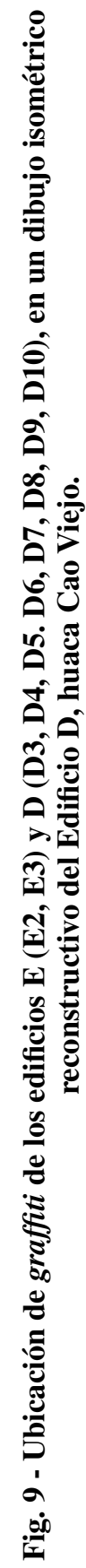




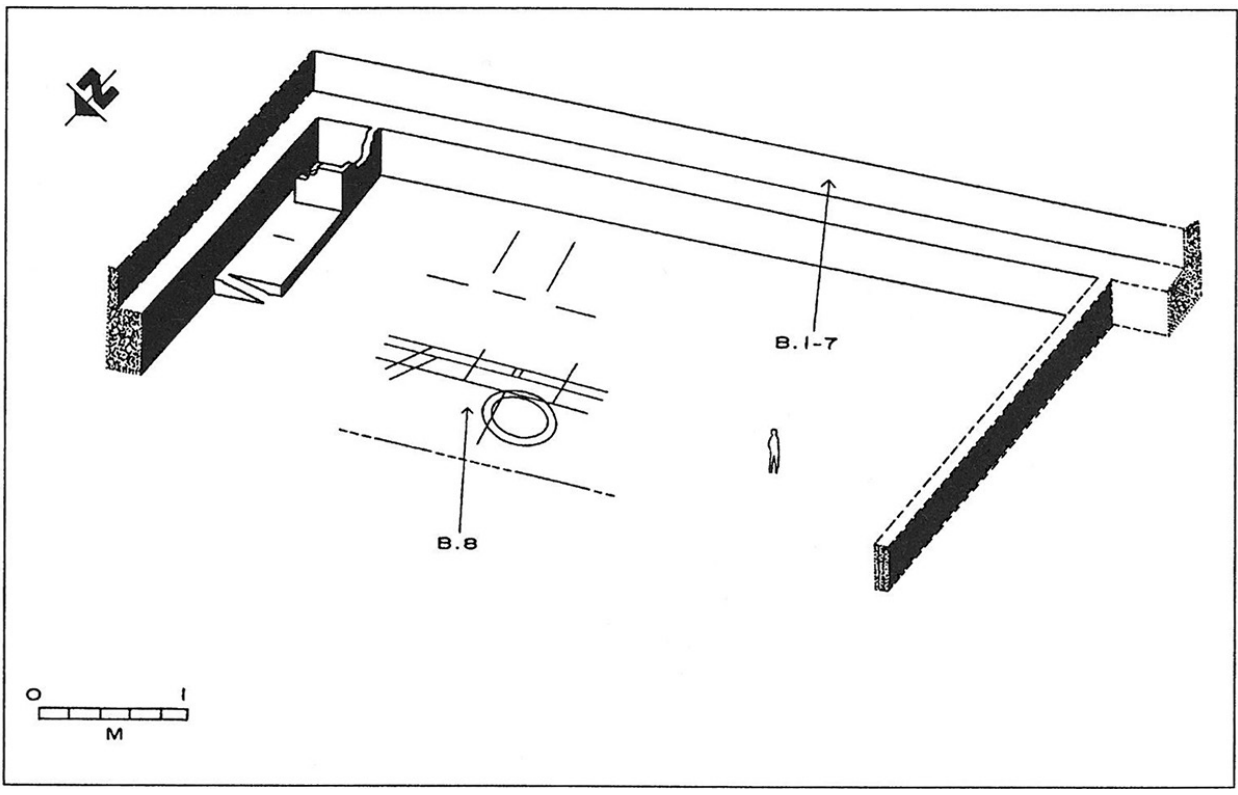

Fig. 10 - Ubicación de graffiti en un dibujo reconstructivo parcial del frontis norte del edificio B, huaca Cao Viejo.

- Localización

- Dimensiones del diseño

- Descripción del (de los) motivo (s).

\section{1. Edificio E}

\begin{tabular}{|llll|}
\hline Código & \multicolumn{1}{c|}{ Localización } & Dimensiones & \multicolumn{1}{c|}{ Descripción } \\
\hline E1 & $\begin{array}{l}\text { Paramento oeste de un muro } \\
\text { ubicado en la esquina NE de la } \\
\text { pirámide, por debajo del piso de la } \\
\text { plaza ceremonial Ancho: } 7,2 \mathrm{~cm} .\end{array}$ & $\begin{array}{l}\text { Altura: } 1,5 \mathrm{~mm} . \\
\text { Cabeza de pez }\end{array}$ & $\begin{array}{l}\text { (life) con apéndices que } \\
\text { se proyectan de la boca. } \\
\text { Las incisiones tienen } \\
\text { restos de pintura negra } \\
\text { (Fig. 4B, 11) }\end{array}$ \\
E2 & $\begin{array}{l}\text { Superficie norte de la segunda } \\
\text { columna (de Este a Oeste), en la } \\
\text { esquina SEdel Patio Ceremonial, }\end{array}$ & Altura: $19 \mathrm{~cm}$. & $\begin{array}{l}\text { Diseño romboidal con } \\
\text { volutas en los vértices; } \\
\text { Plataforma Superior }\end{array}$ \\
& & $\begin{array}{l}\text { en interior se observa } \\
\text { una cruz y círculos; dos } \\
\text { líneas dividen el diseño } \\
\text { en cuatro campos. A un } \\
\text { costado, una figura en } \\
\text { forma de pie (Fig. 4c). }\end{array}$ \\
\end{tabular}


E3 Superficie s ur de la segunda columna (de Este a Oeste), en la

Ancho: $18,5 \mathrm{~cm}$. Tres caras humanas esquina SE del Patio Ceremonial, Altura: $46,5 \mathrm{~cm}$. estilizadas, una de las Plataforma Superior cuales lleva un tocado simple. Se asocian a un conjunto de líneas (Fig. $4 \mathrm{D}, 12)$.

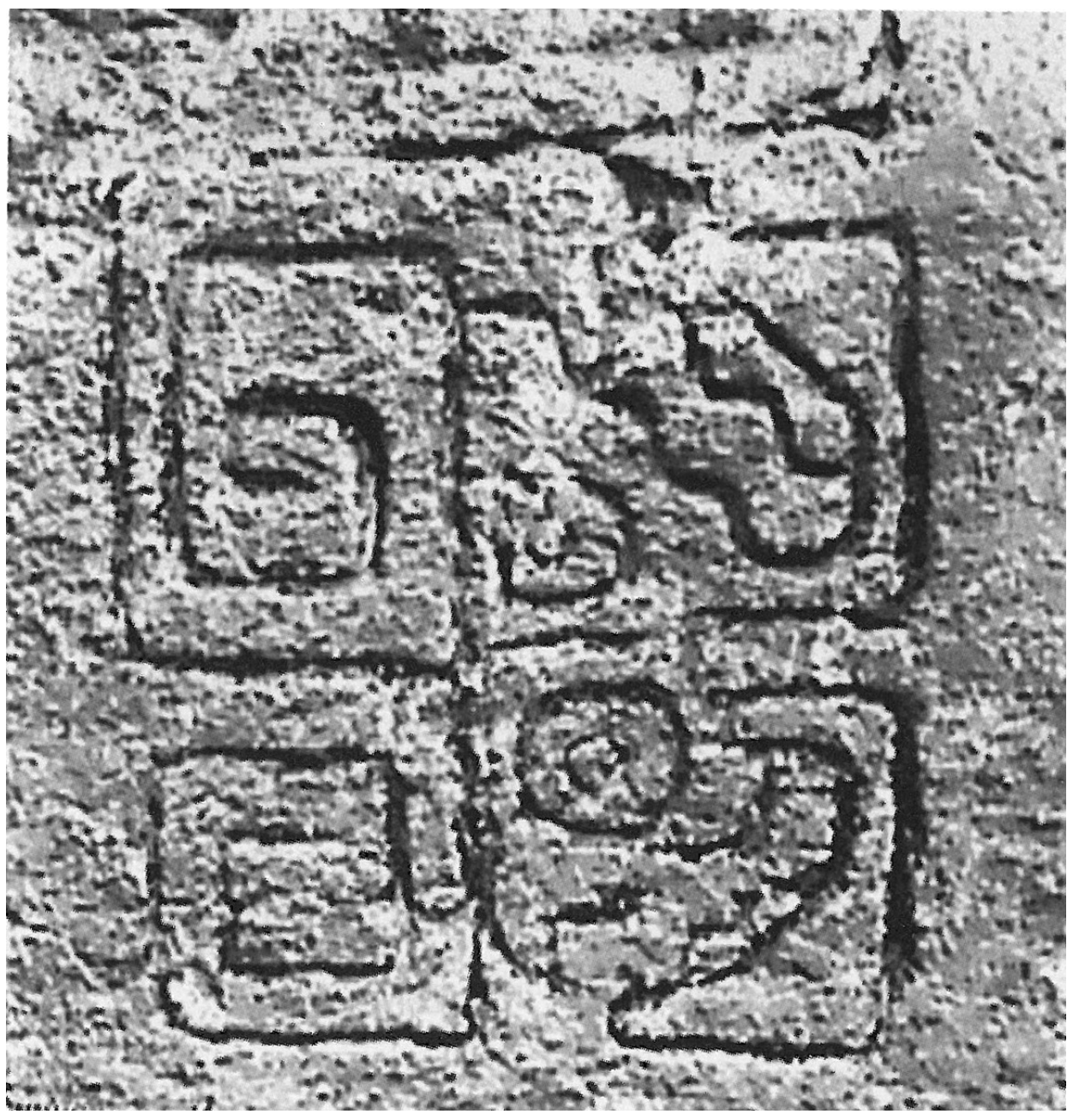

Fig. 11 - Cabeza estilizada de pez cuyas incisiones presentan pintura negra (Diseño E1; ver Fig. 4B). 


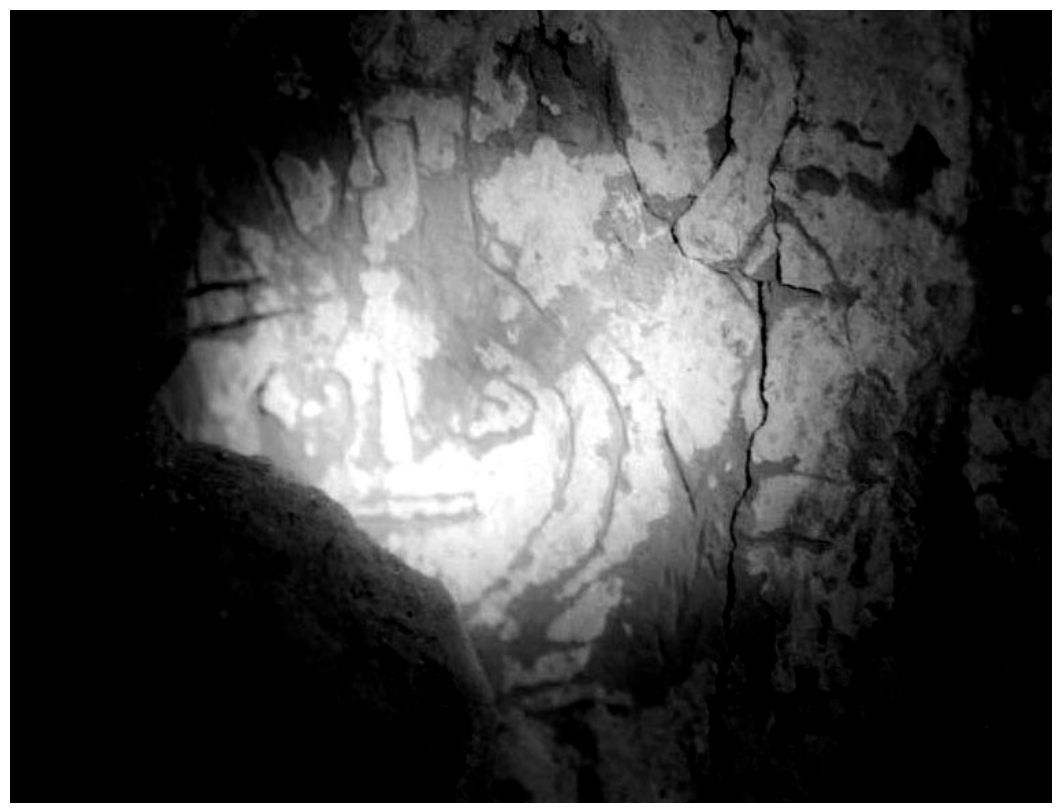

Fig. 12 - Cabeza humana con tocado (detalle del diseño E3, ver Fig. 4D).

\section{2. Edificio D}

\begin{tabular}{|ll|}
\hline Código & Localización \\
\hline D1 & $\begin{array}{l}\text { Superficie sur de una columna } \\
\text { pintada de blanco, en el sector } \\
\text { lateral este de la plaza }\end{array}$ \\
\hline
\end{tabular}

D2 Superficie oeste pintada de rojo de una columna, en el sector lateral este de la plaza

\section{Dimensiones Descripción}

Ancho: $33 \mathrm{~cm}$. Personaje estilizado, Altura: $115 \mathrm{~cm}$. motivo ornitomorfo y otro a manera de porra, asociados a un conjunto de líneas (Fig. 13A)

Ancho: $36 \mathrm{~cm}$. Personaje rodeado de Altura: $92 \mathrm{~cm}$. un conjunto de líneas (Fig. 13B)

D3 Paramento exterior norte del recinto 8, en el Patio Ceremonial, Plataforma Superior. El diseño se ubica en el panel este, debajo de la mano derecha del Decapitador

Ancho: $10,5 \mathrm{~cm}$. Personaje con tocado Altura: $15,5 \mathrm{~cm}$. de perfil mirando hacia el Oeste. El cuerpo no está bien definido. Se observa una cabeza de-lante del personaje (Fig. 14A, 15)

D4 Paramentonortedel recinto 8, Ancho: $9 \mathrm{~cm}$. Cuatromotivos separaen el Patio Ceremonial, Altura: $20,5 \mathrm{~cm}$. dos: una cabeza trofeo 


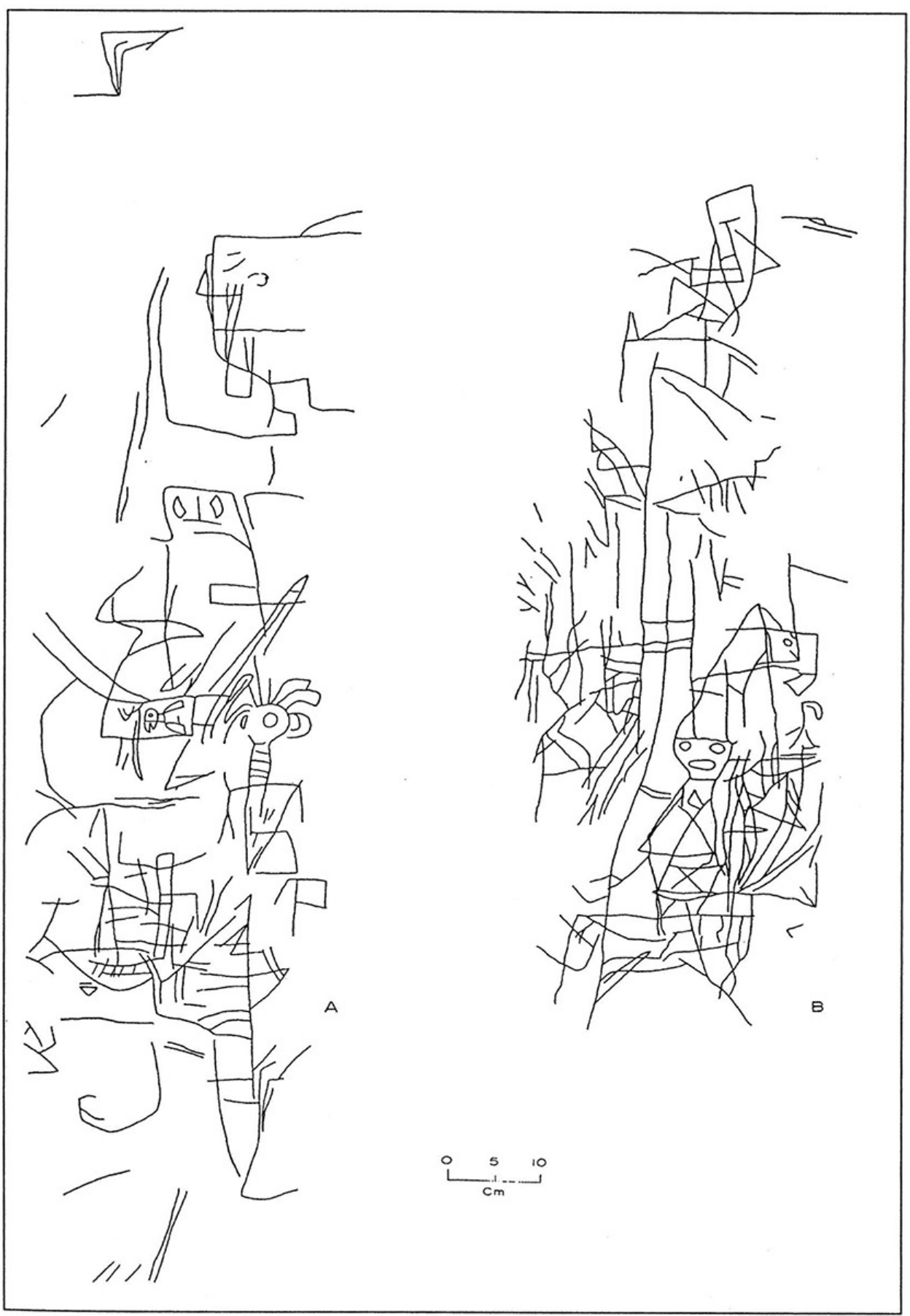

Fig. 13 - Graffiti del sector lateral este de la plaza del edificio D: D1 (A) D2 (B). 


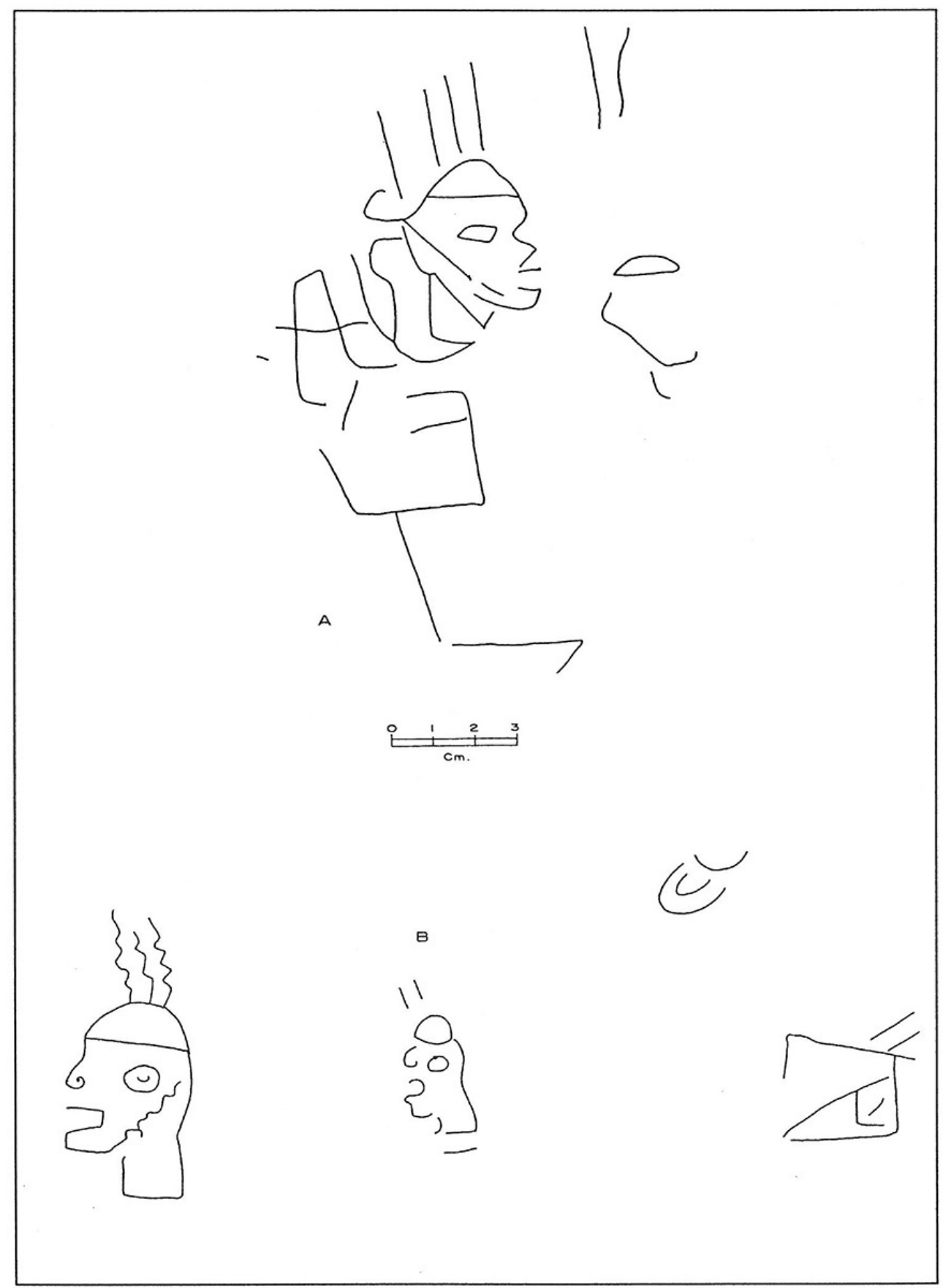

Fig. 14 - Graffiti del recinto 8 (Plataforma Superior) del edificio D: D3 (A), D4 (B). 
Plataforma Superior. El diseño se ubica en el panel este, a la izquierda de la cabeza trofeo sostenida por el Decapitador mirando hacia el Este, que parece imitar la cabeza llevada en la mano izquierda por el Decapitador del mural; un motivo de menor dimensión similar al anterior, tres líneas curvas y una figura geométrica (Fig. 14B, 16)

D5 Paramento interior sur del Recinto 5, sector suroeste de la Plataforma Superior

Ancho: 3,7 cm. Cabeza incompleta de Altura: $6 \mathrm{~cm}$. perfil, con tocado y orejera, mirando hacia el Este (Fig. 17A)

D6 Paramento sur del Recinto 5, sector suroeste de la Plataforma Superior

D7 Paramento interior sur del Recinto 5, sector suroeste de la Plataforma Superior

D8 Paramento interior norte del Recinto 5, sector suroeste de la Plataforma Superior

D9 Paramento interior norte del Recinto 8, Plataforma Superior

D10 Paramento interior oeste del Recinto 8, Plataforma Superior
Ancho: $2,5 \mathrm{~cm}$. Rostro humano inAltura: $2,5 \mathrm{~cm}$. completo (Fig. 17B)

Ancho: $7,5 \mathrm{~cm}$. Motivo indeterminado Altura: $13 \mathrm{~cm}$. (Fig. 17C)

Ancho: $18 \mathrm{~cm}$. Cuchilloceremonialen Altura: 20,5 cm. posicióninvertida(Fig. 17D)

Ancho: $8 \mathrm{~cm}$. Motivos geométricos Altura: $27 \mathrm{~cm}$. diversos (Fig. 17E)

Ancho: $0,7 \mathrm{~cm}$. Diseño reticulado en Altura: $38 \mathrm{~cm}$. posición vertical, insinuando una red de pescar (Fig. 17F)

\section{3. Edificio B}

\begin{tabular}{|c|c|c|c|}
\hline Código & Localización & Dimensiones & Descripciones \\
\hline B1 & $\begin{array}{l}\text { Extremo este de un paramento } \\
\text { del frontis norte de la pirámide }\end{array}$ & $\begin{array}{l}\text { Ancho: } 5 \mathrm{~cm} . \\
\text { Altura: } 7 \mathrm{~cm} .\end{array}$ & $\begin{array}{l}\text { Búho, cuyo cuerpo está } \\
\text { comprimido en sentido } \\
\text { vertical (Fig. 17G) }\end{array}$ \\
\hline B2 & A $2,50 \mathrm{~m}$. al oeste de B1 & $\begin{array}{l}\text { Ancho: } 108 \mathrm{~cm} . \\
\text { Altura: } 96,5 \mathrm{~cm} .\end{array}$ & $\begin{array}{l}\text { Dos probables repre- } \\
\text { sentaciones antro- } \\
\text { pomorfas asociadas a un } \\
\text { conjunto de diseños } \\
\text { indeterminados (Fig. 18) }\end{array}$ \\
\hline
\end{tabular}




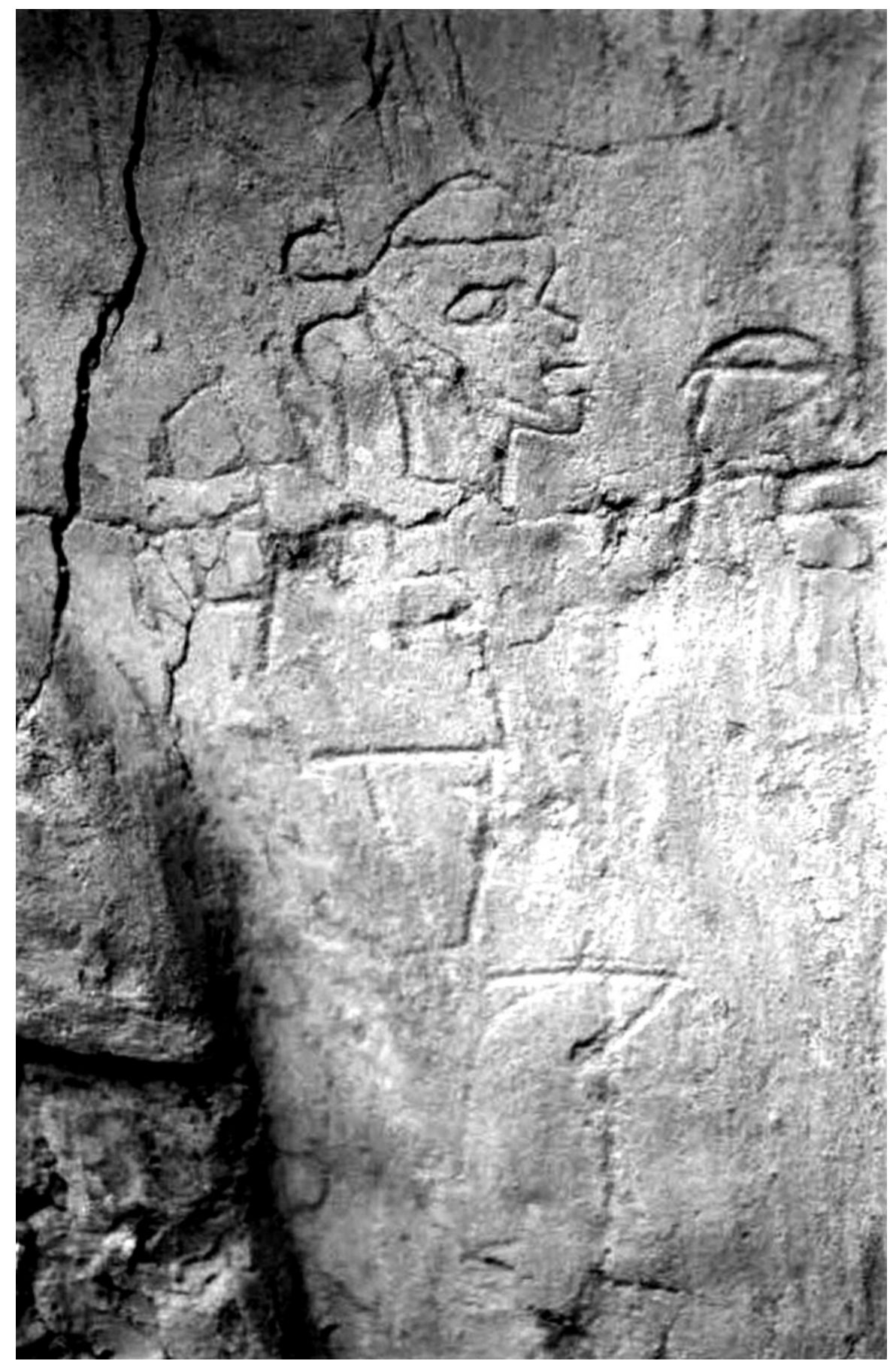

Fig. 15 - Personaje con tocado y esbozo de cabeza humana (Diseño D3, fig. 7A). 


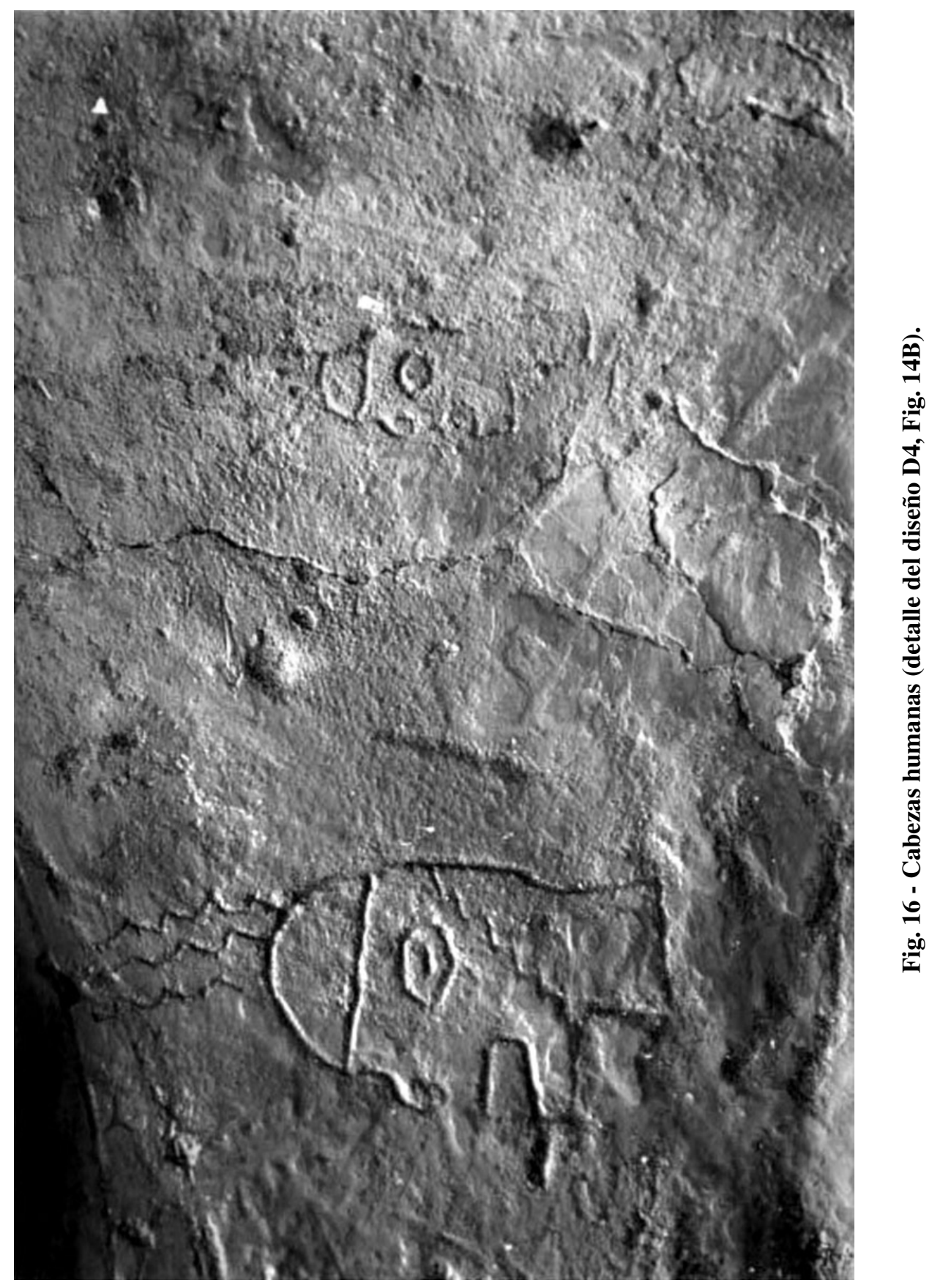




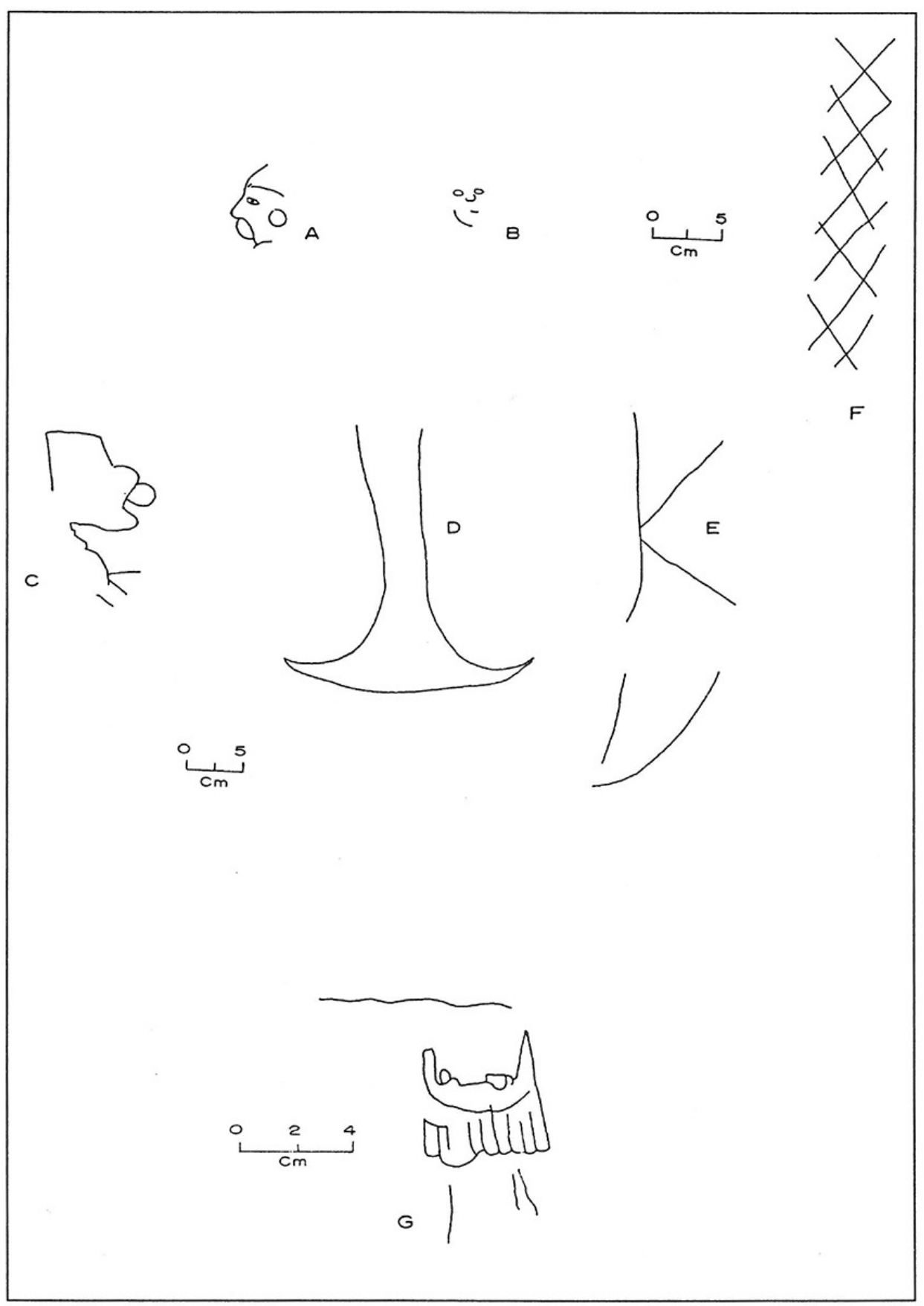

Fig. 17 - Graffiti del recinto 5 (Plataforma Superior) del edificio D: D5 (A), D6 (B), D7 (C), D8 (D), D9 (E), D10 (F); frontis norte del edificio B: B1 (G). 


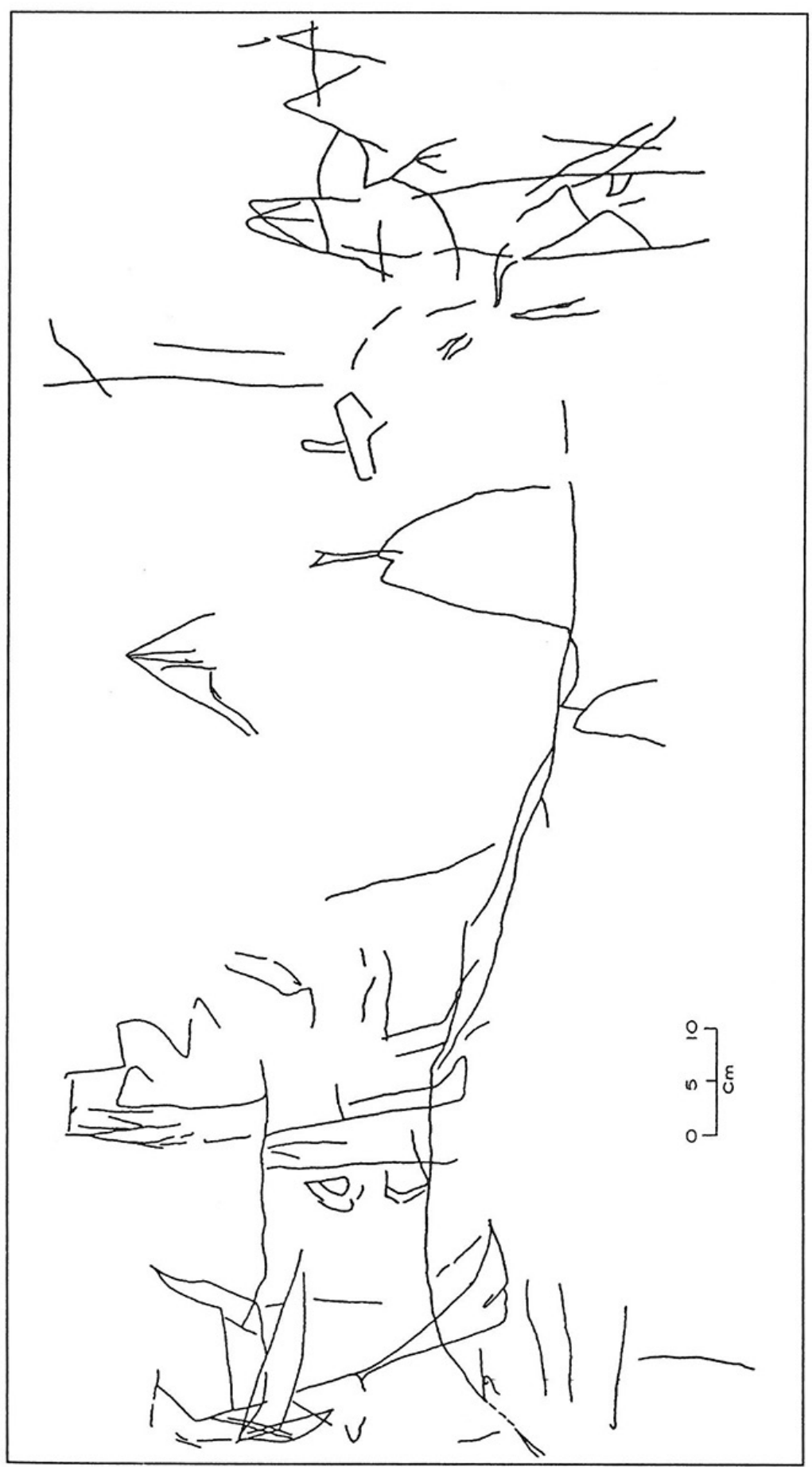

فำ 
B3 A 1,15 m. hacia el oeste de B2 Ancho: 8,5 cm. Extremidades infeAltura: $12 \mathrm{~cm}$. riores orientadas al Oeste, de un cuerpo humano parcialmente destruido por el desmontamiento del muro soporte (Fig. 19A)

B4 A 2 m. hacia el oeste de B3

Ancho: $32 \mathrm{a} 41 \mathrm{~cm}$. Diseños separados por Altura: $27,5 \mathrm{~cm}$. una línea horizontal: figura serpentiforme (parte inferior), bandas curvas superpuestas con círculos (¿cuerpo de serpiente?), asociados a trazos lineales (Fig. 19B)

B5 A $70 \mathrm{~cm}$. hacia el oeste de B4 Ancho: $11 \mathrm{~cm}$. Re presentación Altura: $31 \mathrm{~cm}$. antropozoomorfa de perfil, orientada hacia el Oeste, con rasgos de pez o de reptil. Está sobre un diseño a modo de plataforma (Fig. 19C, 20)

B6 A 1,10 m. hacia el oeste de B5 Ancho: $34 \mathrm{~cm}$. Posiblepiernahumana Altura: $16 \mathrm{~cm}$. orientadahaciaelEste, asociada a líneas que se intersectan (Fig. 19D)

B7 A 9,50m. al oeste de B6 Ancho: 19,5 cm. Partes de una vesAltura: $38 \mathrm{~cm}$. timenta: unku (parte superior) y tocado a manera de casco (parte inferior) (Fig. 19E)

\section{4. Edificio A}

\begin{tabular}{|c|c|c|c|}
\hline Código & Localización & Dimensiones & Descripción \\
\hline A1 & $\begin{array}{l}\text { Banda roja que delimita al Tema } \\
\text { Complejo } 1 \text { por el lado oeste. } \\
\text { Muro norte del Recinto } 1 .\end{array}$ & $\begin{array}{l}\text { Ancho: } 20,5 \mathrm{~cm} . \\
\text { Altura: } 16 \mathrm{~cm} .\end{array}$ & $\begin{array}{l}\text { Guerrero de perfil } \\
\text { orientado hacia elEste, } \\
\text { portando porra y otras }\end{array}$ \\
\hline
\end{tabular}




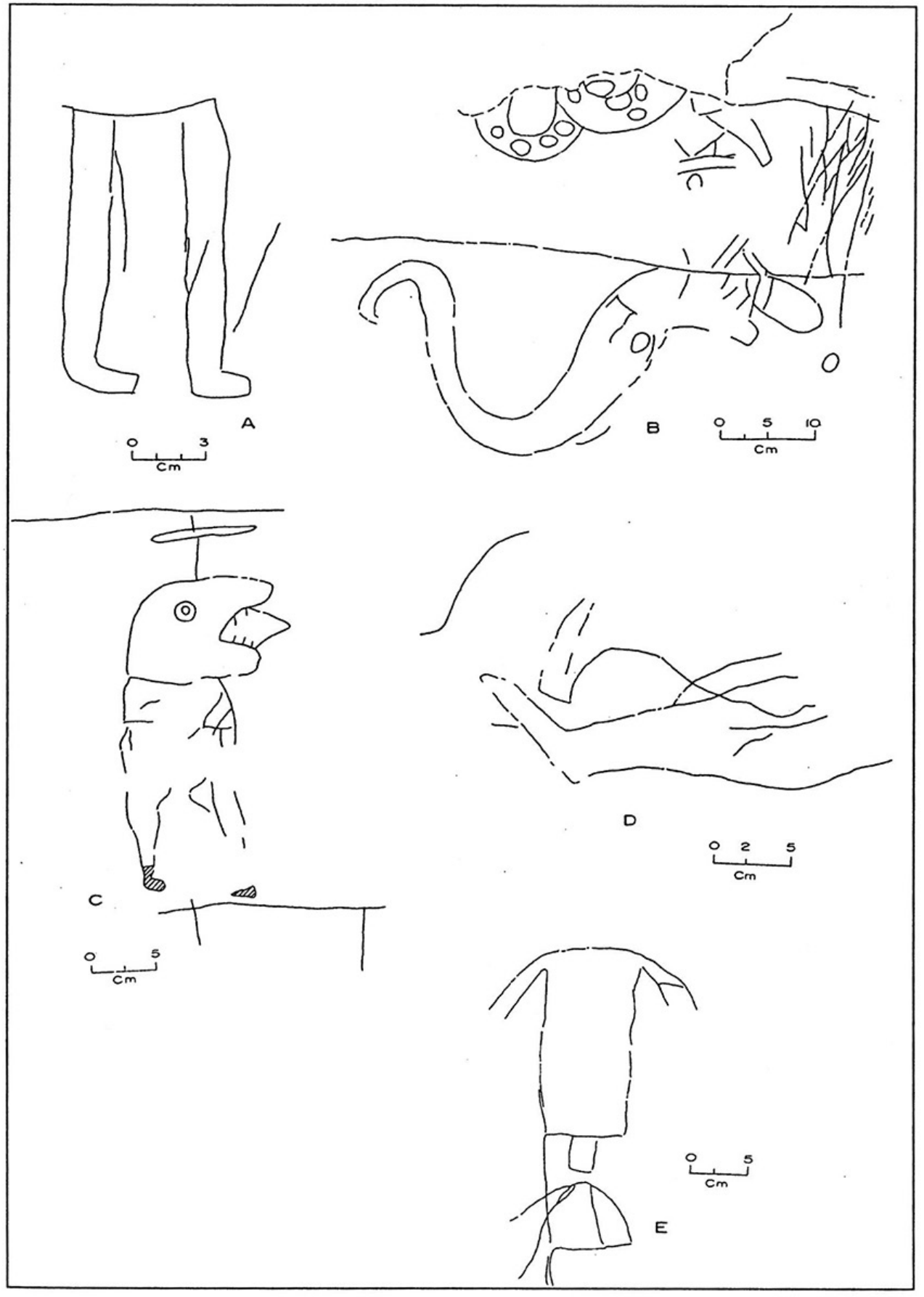

Fig. 19 - Graffiti del frontis norte del edificio B: B3 (A), B4 (B), B5 (C), B6 (D), B7 (E). 


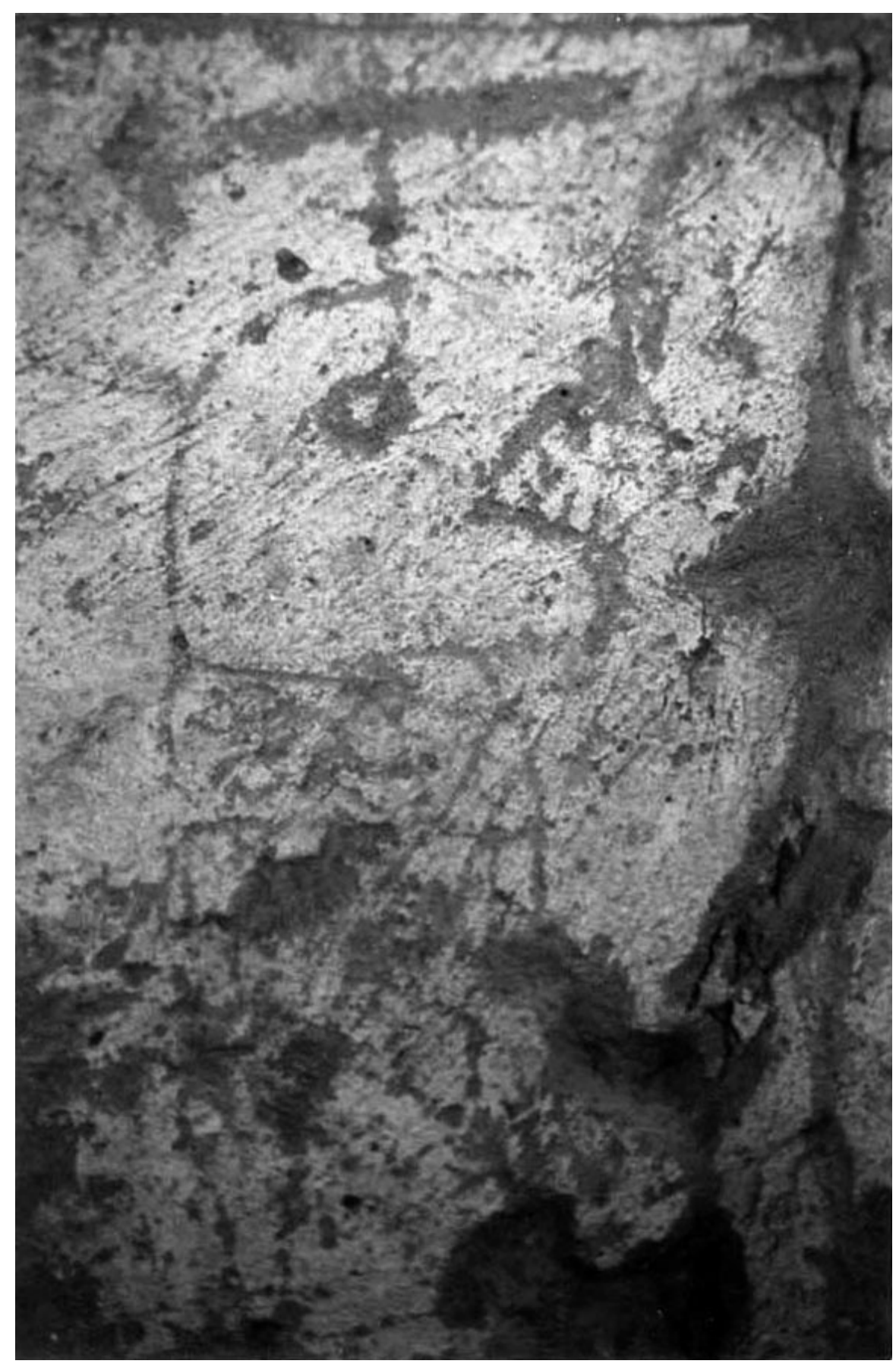

Fig. 20 - Ser antropozoomorfo con rasgos de pez o reptil (diseño B5; Fig. 9C). 
A2 Misma ubicación

Ancho: $9 \mathrm{~cm}$. Motivo zoomorfo. Altura: $15,5 \mathrm{~cm}$. Junto a la cola existe un diseño en forma de caracol. Hay restos de pintura roja en las incisiones (Fig. 6B, 7). Imita uno de los motivos de la Escena de Combate (muro o este del Recinto 1)

A3 Cuarta banda roja horizontal (inferior) de la Escena de Combate. Paramento oeste del Recinto 1.

Ancho: $9 \mathrm{~cm}$. Figura antropomorfa Altura: $6,5 \mathrm{~cm}$. de perfil (incompleta) que mira hacia el Norte. Presenta restos de pintura roja (Fig. 6C)

A4 Paramento con procesión de prisioneros y guerreros. Entre el primer guerrero del lado este y el primer prisionero (debajo del brazo de este último)

A5 Debajo de A4, a la altura de la cintura del primer prisionero

Ancho: $31 \mathrm{~cm}$. Diseño de red, en Altura: $19,5 \mathrm{~cm}$. posición vertical, con flotadores y pesas (Fig. 6E, 21)

A6 Entre las piernas del tercer prisionero

Ancho: $13 \mathrm{~cm}$. Red de pesca, con Altura: $13 \mathrm{~cm}$. floradores y pesas (Fig. $6 \mathrm{D}, 21)$

Ancho: $15,5 \mathrm{~cm}$. Motivo incompleto en Altura: $16 \mathrm{~cm}$. doble escalón (Fig. 6F, 22)

A7 Entre el cuarto y quinto prisionero

Ancho: $12,5 \mathrm{~cm}$. Pez con la cabeza Altura: $6,5 \mathrm{~cm}$. orientada al Oeste. Presenta cuatro líneas sobre la cabeza. Tiene pintura blanca en las incisiones (Fig. $8 \mathrm{~A}$ ).

A8 Entre el cuarto y el quinto prisionero, a la altura de los pies

Ancho: $30 \mathrm{~cm}$. Motivo indefinido Altura: $22,5 \mathrm{~cm}$. sobre un triángulo(Fig. 8B)

A9 Entre las piernas del quinto prisionero

Ancho: $11 \mathrm{~cm}$. Motivo antropomorfo Altura: $23 \mathrm{~cm}$. esquematizado en posi- 


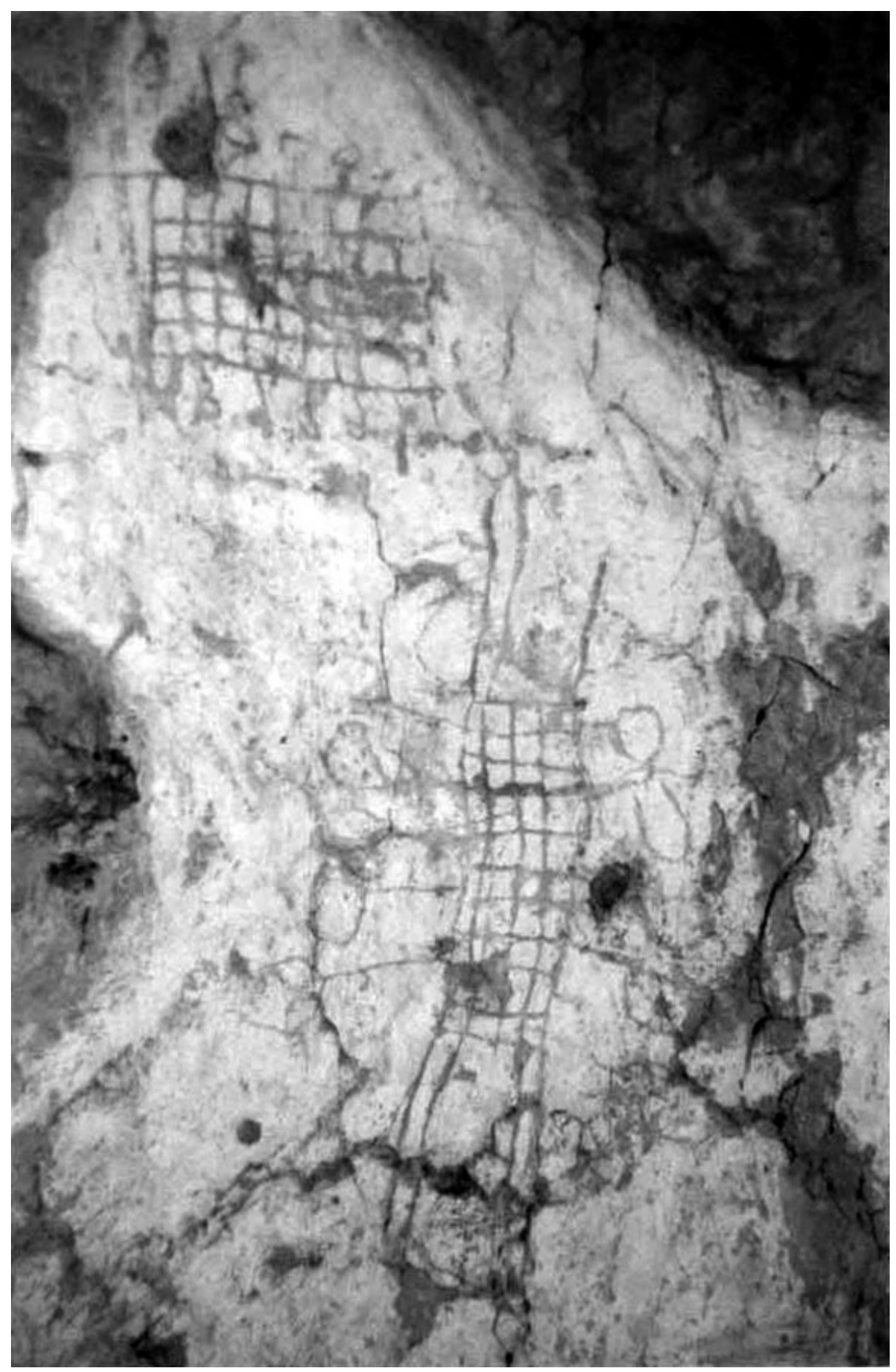

Fig. 21 - Redes de pescar (diseños A4, superior, y A5, inferior) (ver Fig. 6D y 6E). 


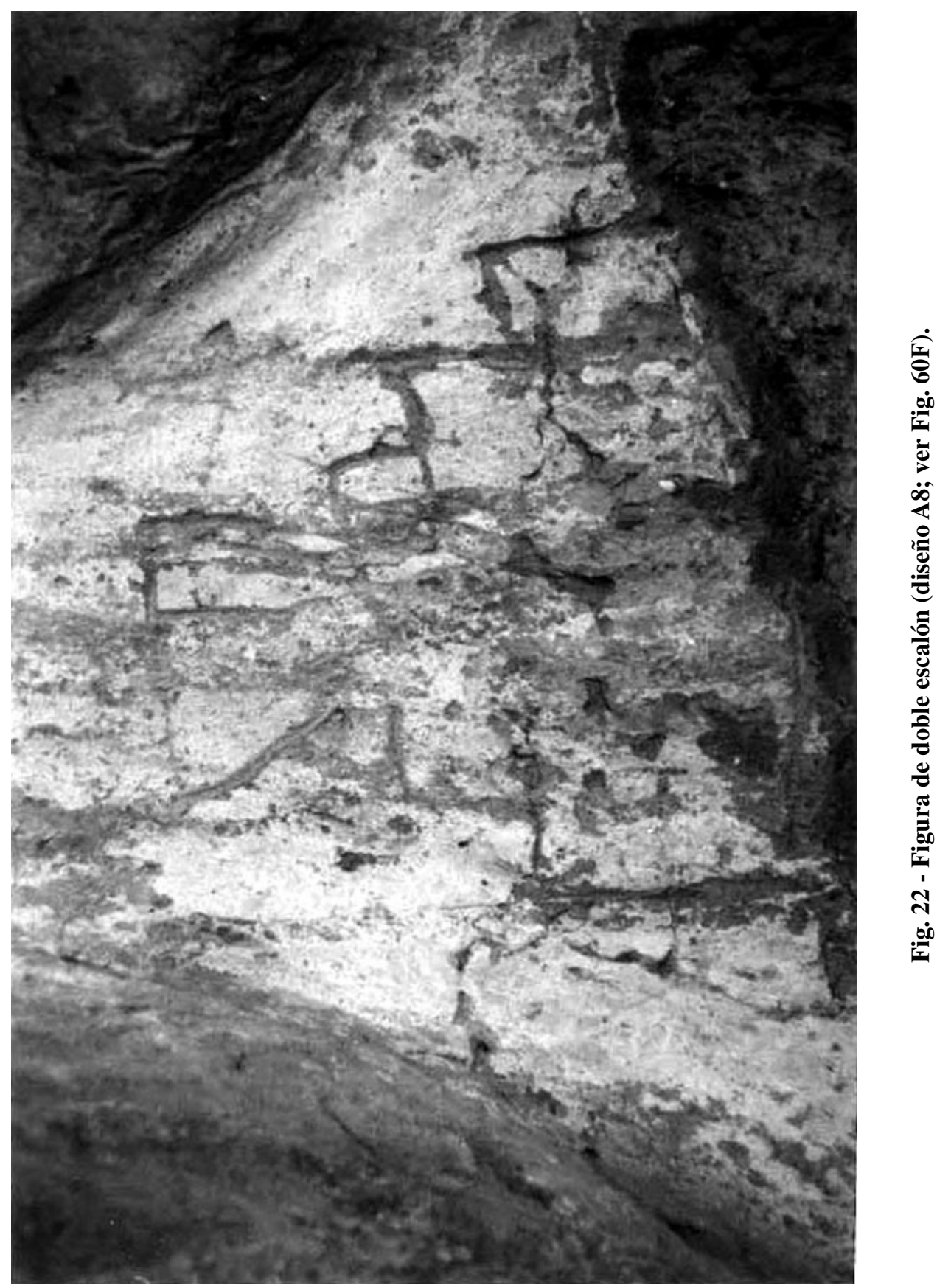


ción frontal, con un apéndice en la cabeza a mododetocado(Fig.8C)

A10 Entre el sexto y el séptimo prisionero

A11 Entre el octavo y noveno prisioneros

Ancho: $16 \mathrm{~cm}$. Diseño indeterminado Altura: $20 \mathrm{~cm}$. (Fig. 8D)

Ancho: $31 \mathrm{~cm}$. Motivo antropomorfo Altura: $15 \mathrm{~cm}$. de cuerpo alargado, asociado a un conjunto de líneas sinuosas (Fig. 8E)

A12 En la pierna derecha, pintada de amarillo, del tercer guerrero ubicado detrás del último prisionero

Ancho: $28 \mathrm{~cm}$. Motivos estilizados: Altura: $41 \mathrm{~cm}$. diseño reticulado al interior de un conjunto de líneas. Las incisiones tienen pintura ocre (Fig. 8F)

A13 En la pierna izquierda, pintada de amarillo, del mismo guerrero

Ancho: $16 \mathrm{~cm}$. Altura: $31 \mathrm{~cm}$.

Re presentación zoomorfa (¿zorro?) en posición vertical sobre una línea que se intersecta con otras. En la parte superior hay un diseño semicircular. Las incisiones tienen pintura amarilla (Fig. 8G)

A14 Extremo oeste del paramento con la procesión de prisioneros y guerreros, y entre las piernas de dos guerreros

Ancho: $9,5 \mathrm{~cm}$. Motivo antropomorfo Altura: $7,6 \mathrm{~cm}$. de perfil orientado hacia el Oeste, que lleva una porra $y$ posiblemente un escudo (Fig. 23A)

A15 Paramento oeste del tema de los Personajes Asidos de la Mano, y entre las cabezas del primer y segundo personaje (de Sur a Norte)

A16 Mismo paramento. Entre el octavo y noveno prisioneros

Ancho: $8 \mathrm{~cm}$. Dos motivos trianAltura: $14 \mathrm{~cm}$. gulares (Fig. 23C)

A17 Paramento interior oeste del Ancho: $92 \mathrm{~cm}$. Posiblerepresentación Recinto 1

Altura: $69 \mathrm{~cm}$. antropomorfa con tocado. A la izquierda se 


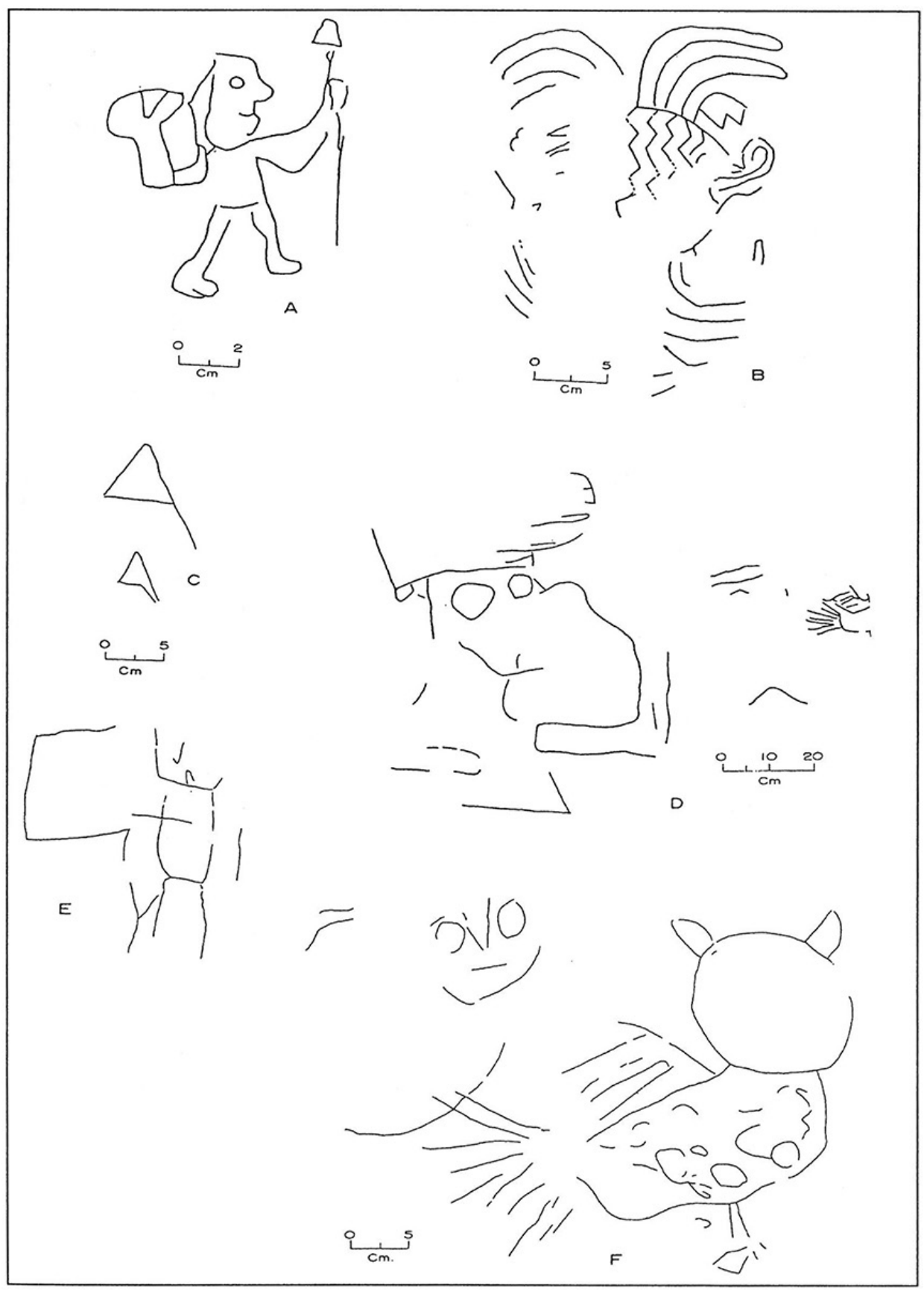

Fig. 23 - Graffiti del edificio A, frontis norte: procesión de prisioneros y guerreros: A14 (A), A15 (B), A16 (C); interior del Recinto 1: A17 (D), A18 (E), A19 (F). 


\begin{tabular}{|c|c|c|c|}
\hline & & & $\begin{array}{l}\text { insinúa la cola de un } \\
\text { ave (Fig. 23D) }\end{array}$ \\
\hline A18 & Mismo paramento & $\begin{array}{l}\text { Ancho: } 19 \mathrm{~cm} . \\
\text { Altura: } 20 \mathrm{~cm} .\end{array}$ & $\begin{array}{l}\text { Motivo geométrico } \\
\text { indeterminado (Fig. } \\
\text { 23E) }\end{array}$ \\
\hline A19 & $\begin{array}{l}\text { Paramento interior sur del } \\
\text { Recinto } 1\end{array}$ & $\begin{array}{l}\text { Ancho: } 46 \mathrm{~cm} . \\
\text { Altura: } 35 \mathrm{~cm} .\end{array}$ & $\begin{array}{l}\text { Búho de perfil, } \\
\text { mirandohacia el Norte. } \\
\text { Sobre la cola se insinúa } \\
\text { en forma simplificada } \\
\text { otro búho (Fig. 23F, } \\
24 \text { ) }\end{array}$ \\
\hline A20 & Mismo paramento & $\begin{array}{l}\text { Ancho: } 83 \mathrm{~cm} . \\
\text { Altura: } 56 \mathrm{~cm} .\end{array}$ & $\begin{array}{l}\text { Búho en posición } \\
\text { frontal, con las alas } \\
\text { desplegadas ymirando } \\
\text { hacia el Norte (Fig. } \\
\text { 25A) }\end{array}$ \\
\hline A 21 & $\begin{array}{l}\text { Paramento interior oeste del } \\
\text { Recinto } 4 \text {, sector exterior oeste } \\
\text { de la plaza }\end{array}$ & $\begin{array}{l}\text { Ancho: } 21 \mathrm{~cm} . \\
\text { Altura: } 30 \mathrm{~cm} .\end{array}$ & $\begin{array}{l}\text { Personaje incompleto } \\
\text { de perfil, que lleva un } \\
\text { tocado. Está rodeado } \\
\text { por líneas diversas y } \\
\text { un motivo escalonado } \\
\text { (Fig. 25B) }\end{array}$ \\
\hline A22 & Mismo paramento & $\begin{array}{l}\text { Ancho: } 53 \mathrm{~cm} . \\
\text { Altura: } 73 \mathrm{~cm} .\end{array}$ & $\begin{array}{l}\text { Personaje de perfil } \\
\text { mirando hacia el } \\
\text { Norte. De su tocado, } \\
\text { pende una larga trenza } \\
\text { rematada en una } \\
\text { cabeza zoomorfa. } \\
\text { Posiblemente lleva } \\
\text { escudoy otros objetos. } \\
\text { Enellado sur se asocia } \\
\text { a una representación } \\
\text { estilizada (Fig. 25C) }\end{array}$ \\
\hline A 23 & $\begin{array}{l}\text { Muro sur de la Plataforma } \\
\text { Principal, asociado a los } \\
\text { relieves polícromos en rombos }\end{array}$ & $\begin{array}{l}\text { Ancho: } 17 \mathrm{~cm} \\
\text { Altura: } 10 \mathrm{~cm} .\end{array}$ & $\begin{array}{l}\text { Motivo en forma de } \\
\text { espiga (Fig. 25D) }\end{array}$ \\
\hline
\end{tabular}

\section{COMENTARIO}

El tratamiento del tema de los graffiti mochicas no ha sido exhaustivamente abordado, debido a que hasta fines de la década del 80 no se habían realizado trabajos de largo alcance en los edificios monumentales. Por esta razón, los datos que reportamos requieren ser contrastados con otros hallazgos aún inéditos en la costa norte del Perú, 


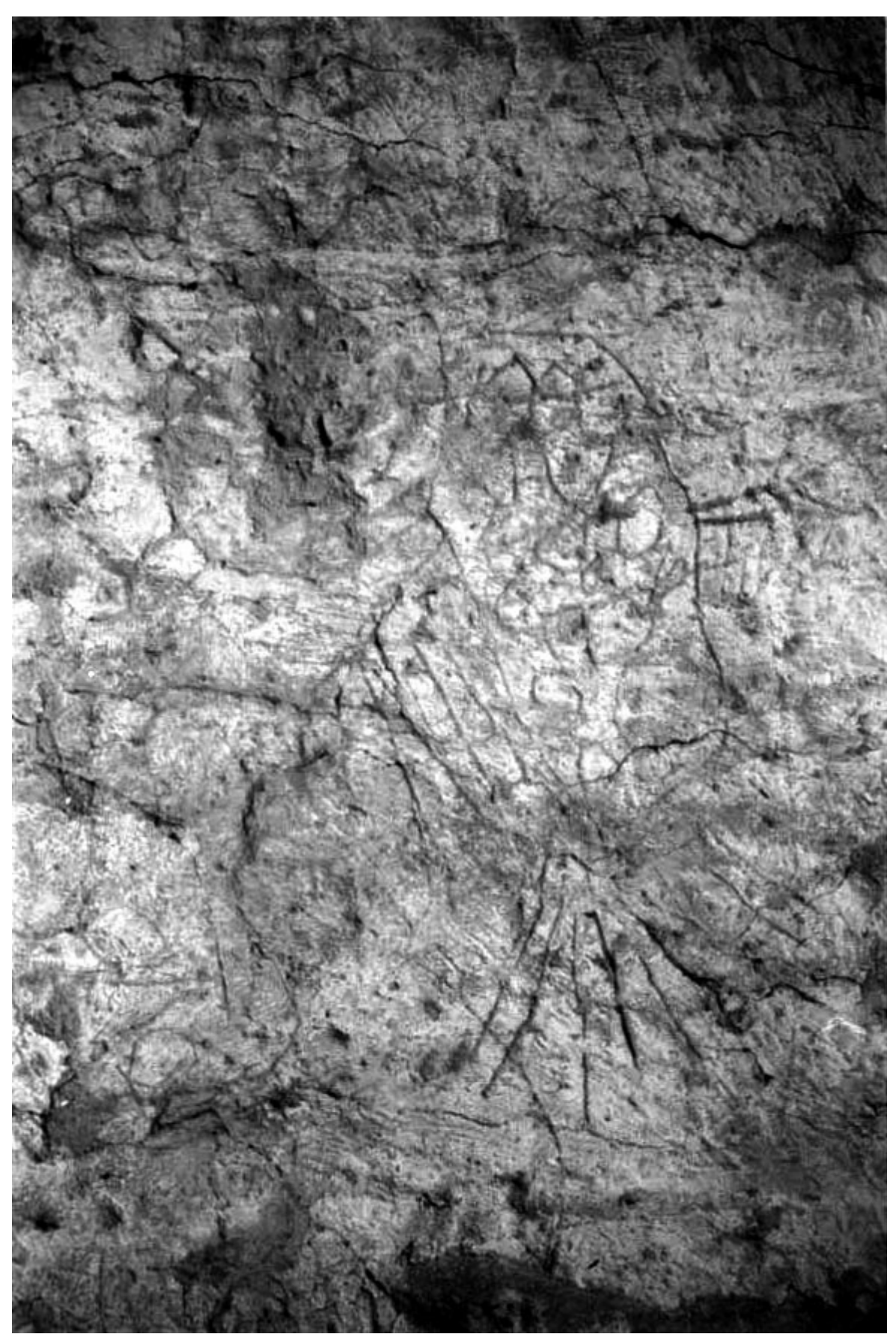

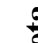

흠

$\underline{9}$

葛

8

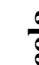

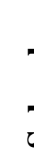

.

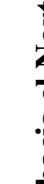

ปั. 


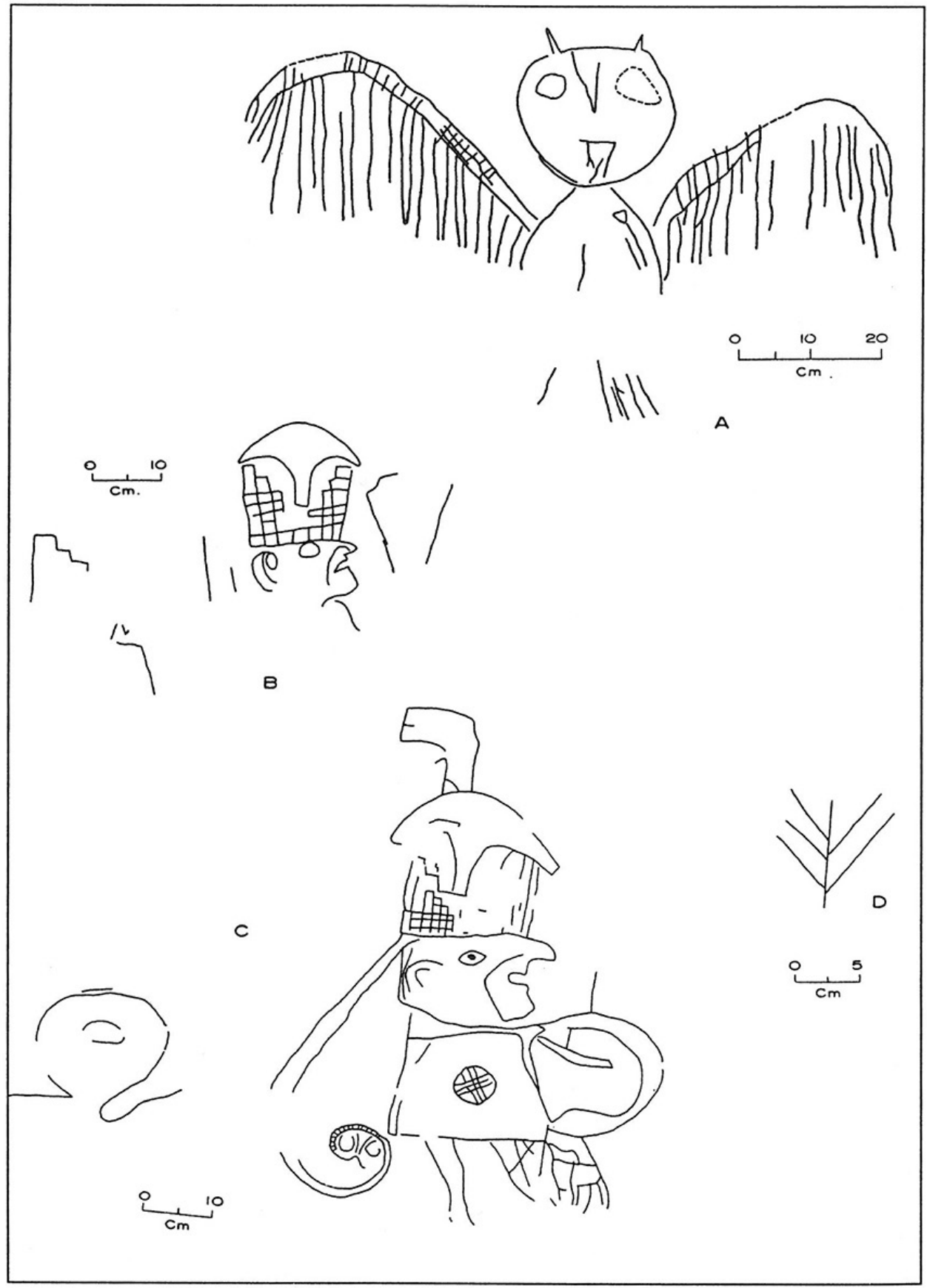

Fig. 25 - Graffiti del interior del recinto 1 del edificio A: A20 (A); interior del recinto 4, en el sector exterior oeste de la plaza del edificio A: A21 (B), A22 (C); paramento sur de la Plataforma Principal: A23 (D). 
para discutir las respuestas a nuevas interrogantes y enriquecer las interpretaciones que se exponen.

Los graffiti representados en la huaca Cao Viejo y en varios edificios ceremoniales mochicas de la costa norte y norcentral, en muchos casos están asociados, aunque no necesariamente contemporáneos a los temas representados en los relieves polícromos y pintura mural. Se considera que estos temas y posiblemente una parte de los graffiti que se reportan contribuyeron a la definición del carácter de los espacios durante la función de los edificios A, B, D y E, así como después del abandono de éstos (Franco et al., 1994b). Por otra parte, los graffiti también son recurrentes en otros edificios premochicas y post-mochicas de la costa peruana.

Es necesario comentar el planteamiento que hace Alcina Franch (1987) acerca de los graffiti. En nuestra opinión, para el caso de la huaca Cao Viejo y los otros sitios costeños con este tipo de diseños, no estamos de acuerdo que en todos los casos conocidos se trate de "cierto 'arte' inconsistente y trivial... que no es otra cosa como el arte de lo que no tiene intención de serlo" (Alcina Franch, 1987: 161). Por el contrario, existen diseños que reflejan una pre-concepción intelectual y simbólica, pero que, por las circunstancias en que se ejecutan, se reducen a simplificaciones de fácil elaboración, que abrevian símbolos y otros elementos iconográficos que encierran cierto sentido religioso y probablemente una extensión de otros temas pictográficos mochicas.

Sin embargo, hay características (Alcina Franch, 1987) que sí se cumplen en el caso de graffiti existentes en los templos prehispánicos: copismo de los diseños, intención mágica, espontaneidad; pero otros, como la aparente intención no artística, la no especialización, y el que (el "arte") no es contemplativo, no pueden ser generalizados si se considera el corpus de datos que presentamos y aquél que ha sido publicado por los otros autores (Franco et al., 1994b; Bennett, 1950: fig. 4; Ubbelohde-Doering, 1957: 410, fig. 1-3; Franco, 1998b: fig. 49a-c; Donnan, 1972: 88-91; Donnan, 1989: 127; Shimada, 1989: 177-180, fig. 8, 10a, b; Donnan \& Cock, 1997: 21, fig. 10; Uceda \& Mujica, 1994: carátula del libro; Schaedel, 1970: 115).

En el edificio E, los diseños representan motivos poco comunes, salvo la representación geometrizada del pez Trichomycterus sp. ("life"). En el edificio D se presentan, por primera vez, motivos completos de personajes y animales esquematizados, además de la imitación de las cabezas trofeo en miniatura, localizadas dentro del panel este, que contiene el relieve del Decapitador, mientras que otras figuras se componen de trazos geométricos sin orden aparente.

Como las representaciones del edificio B están dentro de un edificio inconcluso, es probable que hayan sido elaboradas en un momento de crisis previo al remodelamiento que dio lugar al edificio A, por lo cual es mucho más difícil determinar la forma de las representaciones y su interpretación. Los graffiti, plasmados sólo en un paramento pintado de blanco del frontis norte de la pirámide, aluden a formas esquematizadas ornitomorfas, antropomorfas e ictiomorfas.

Por último, los graffiti del Edificio A son los más numerosos y tienden a ser figurativos, como lo es el arte mochica en general. Llama la atención los diseños representados en el marco de la esquina noroeste del Recinto Ceremonial por su bien lograda ejecución, entre ellos el guerrero (Fig. 6A, 7) y el felino (Fig. 6B, 7), que imitan 
diseños de la Escena de Combate y el Tema Complejo 1. Asimismo, las redes de pescar (Fig. 6D, 6E, 21), que imitan una figura en relieve del Tema Complejo 1. En este edificio debemos tener en cuenta la relación que existe entre los graffitti y los espacios arquitectónicos ceremoniales.

En el caso del interior del Recinto 1 en la plaza ceremonial, destacan las representaciones del búho, ave nocturna de reconocido simbolismo en la iconografía mochica la cual está ligada al mundo de los muertos y a la oscuridad. También se le podría vincular a las funciones de los personajes con atributos de esta ave nocturna, entre ellas: la actividad de sacrificio de prisioneros (Kutscher, 1983: fig. 267, 273; Bourget, 1996: 39-41; Donnan \& McClelland, 1999: 429). Es sugerente que las representaciones de búhos del Recinto Ceremonial se encuentren en un ambiente pequeño y cerrado.

La gran mayoría de motivos geométricos y estilizados corresponden a abstracciones que fueron complementadas con motivos de corte naturalista, algunos de los cuales copian imágenes existentes en los relieves polícromos. Este es el caso de la araña (Fig. 23B) grabada en el paramento este del tema de los Personajes Asidos de la Mano (Oficiantes), graffiti que se ubica cerca de la figura del Decapitador (Fig. 26), el cual presenta atributos de este artrópodo. Como se sabe, la araña aparece en la iconografía desde la época Cupisnique (Salazar \& Burger, 1981: 241) y, de otro lado, Cordy-Collins (1992) ha comparado a ese animal con los guerreros mochicas en el aspecto de la captura de sus víctimas y la extracción de sus fluidos vitales.

\section{Referencias citadas}

ALCINA FRANCH, J., 1987 - Arte y Antropología; Madrid: Alianza Editorial.

ARÉVALO, W., MILLA, S. \& TORIBIO, R., 1998 - Secuencia constructiva del edificio principal en el Montículo 2, complejo arqueológico El Brujo, valle de Chicama, 75p.; Trujillo: Universidad Nacional de Trujillo. Informe de prácticas pre-profesionales de Arqueología.

BENNETT, W. C., 1950 - The Gallinazo group, Viru Valley, Peru, New Haven: The Museum of Modern Art.

BONAVIA, D., 1974 - Ricchata quellccani. Pinturas murales prehispánicas, 187p., Lima: Fondo del Libro del Banco Industrial del Perú.

BOURGET, S., 1996 - Los raptores de almas: prácticas funerarias en la iconografía mochica. In: Al final del camino (Millones \& Lemlij, eds.): 37-50; Lima. Seminario Interdisplinario de Estudios Andinos.

CAMPANA, C., 1994 - La cultura Mochica, 168p., Lima: CONCYTEC.

CORDY-COLLINS, A., 1992- Archaism or tradition? The decapitation theme in Cupisnique and Moche iconography. Latin American Antiquity, 3 (3): 206-220.

DONNAN, C., 1972 - Moche-Huari nurals from northern Peru. Archaeology, 25(2): 85-95.

DONNAN, C., 1989 - En busca de Naylamp: Chotuna, Chornancap y el valle de Lambayeque. In: Lambayeque, Arte y Tesoros del Perú (Antonio de Lavalle, ed.): 105-135; Lima: Banco de Crédito del Perú.

DONNAN, C. \& McClELlAND, D., 1999 - Moche fineline painting, 319p.; Los Angeles: Fowler Museum of Cultural History, University of California. 


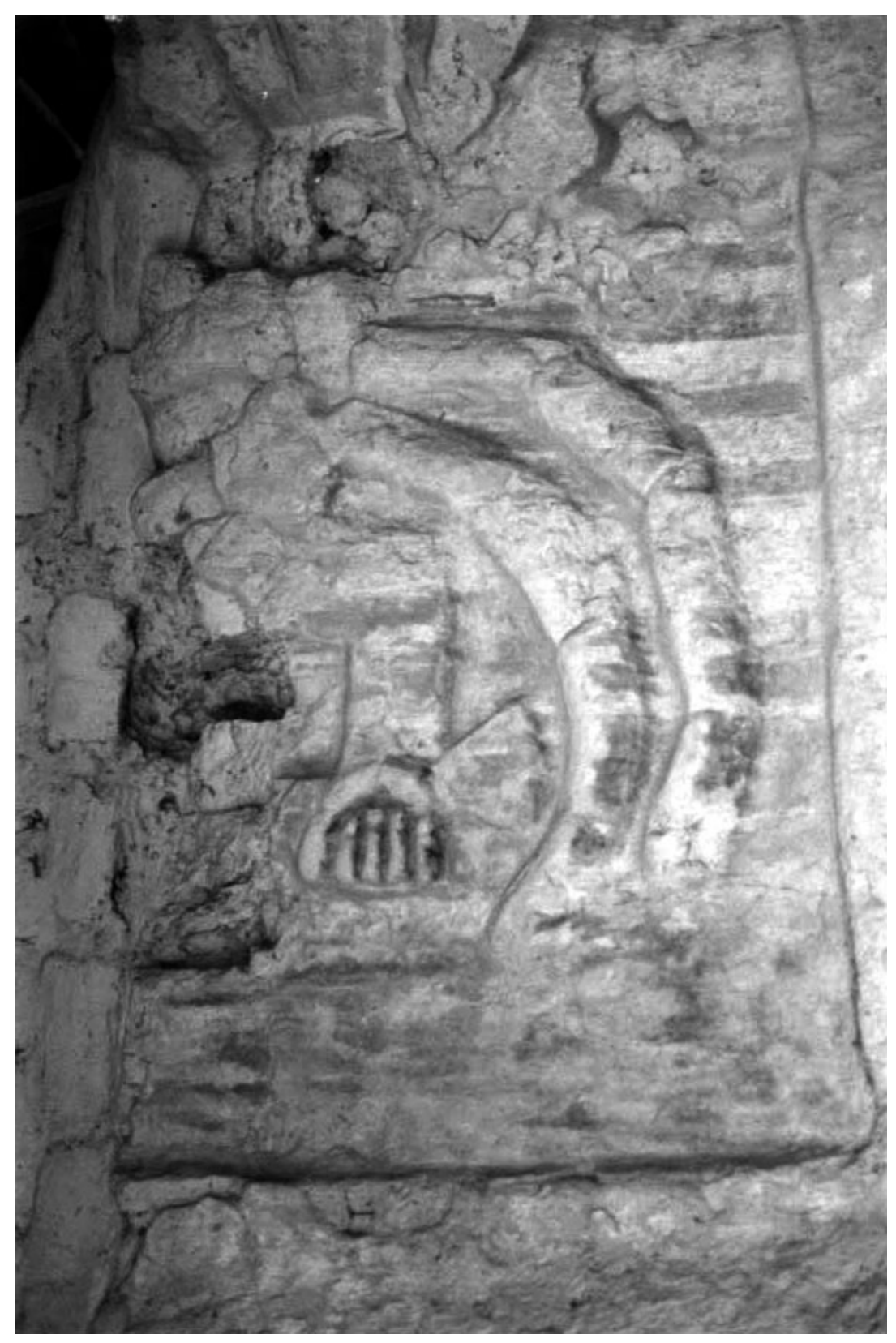

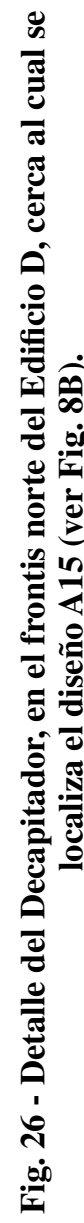


DONNAN, C. \& COCK, G., 1997 - Excavaciones en Dos Cabezas, valle del Jequetepeque, Perú. Tercer Informe Parcial de la Tercera Temporada de Excavaciones (julio-agosto, 1996), presentado al Instituto Nacional de Cultura.

FRANCO, R., 1998a - Arquitectura monumental moche. Correlación y espacios arquitectónicos. Arkinka, 27: 100-110.

FRANCO, R., 1998b - La pirámide con rampa $N^{o} 2$ de Pachacamac. Excavaciones y nuevas interpretaciones, 105p; Trujillo.

FRANCO, R. \& MURGA, A., 1998 - Un modelo arquitectónico de piedra. Medio de Construcción, 133: $16-22$.

FRANCO, R., GÁLVEZ, C. \& VÁSQUEZ, S., 1994a - Arquitectura y decoración Mochica en la Huaca Cao Viejo, complejo El Brujo: Resultados preliminares. In: Moche: Propuestas y perspectivas (Uceda \& Mujica, editores): 147-180; Lima: Universidad de TrujilloIFEA-FOMCIENCIAS. Actas del Primer Coloquio sobre la Cultura Moche 1993. Travaux de l'Institut Francais d'Etudes Andines, 79.

FRANCO, R., GÁLVEZ, C. \& VÁSQUEZ, S., 1994b - Informe final de la temporada 1994, Trujillo: Programa Arqueológico Complejo El Brujo.

FRANCO, R., GÁLVEZ, C. \& VÁSQUEZ, S., 1996 - Los descubrimientos arqueológicos en la Huaca Cao Viejo, complejo El Brujo. Arkinka, 5: 82-94.

FRANCO, R., GÁLVEZ, C. \& VÁSQUEZ, S., 1998a - Desentierro ritual de una tumba moche. Revista Arqueológica Sián, 6: 9-18.

FRANCO, R., GÁLVEZ, C. \& VÁSQUEZ, S., 1998b - Un cielorraso moche polícromo. Medio de Construcción, 144: 37-42.

FRANCO, R., GÁLVEZ, C. \& VÁSQUEZ, S., 1998c - Un pozo ceremonial Moche en el Complejo Arqueológico “El Brujo”. Revista de la Facultad de Ciencias Sociales, 5: 307327; Trujillo: Universidad Nacional de Trujillo.

FRANCO, R., GÁLVEZ, C. \& VÁSQUEZ, S., 1998d-Estado de las investigaciones arqueológicas en la Huaca Cao Viejo, complejo El Brujo, Costa Norte. In: I Encuentro de Peruanistas: 143-160; Lima:Universidad de Lima, Centro de Desarrollo.

FRANCO, R., GÁLVEZ, C. \& VÁSQUEZ, S., 1999a - Tumbas de cámara Moche en la plataforma superior de la Huaca Cao Viejo, complejo El Brujo. Boletín del Programa Arqueológico Complejo “El Brujo”, 1: 1-29.

FRANCO, R., GÁlVEZ, C. \& VÁSQUEZ, S., 1999b - Porras mochicas del Complejo "El Brujo". Revista Arqueológica SIAN, 7: 16-23.

HECKER, G. \& HECKER, W., 1995 - Die Aubgrabungen von Heinrich Ubbelohde - Döering in Pacatnamú, Nord Perú. Untersuchungen Anden Huacas 31 und 14 sowie Bestattungen und Fundobjekte, 497p.; Berlín: Dietrich Reimer Verlag.

HOCQUENGHEM, A. M., 1987 - Iconografía Mochica, 280p., Lima: Fondo Editorial Pontificia Universidad Católica del Perú.

KUTSCHER, G., 1983 - Nordperuanische Gefäßmalereien des Moche-Stils, 320 p., Materialien zur Allgemeinen und Vergleichenden Archäologie 18, Kommision für Allgemeinen und Vergleichenden Archäologie (CAVA); München: Verlag C. H. Beck.

LARCO HOYLE, R. 1938 - Los Mochicas, Tomo 1, 142p; Lima: Editorial Rimac S.A.

SALAZAR, L. \& BURGER, R., 1981 - La araña en la iconografía del Horizonte Temprano en la costa norte del Perú. Beitrage zur Allgemeinen und Vergleinchenden Archäologie, 4: 213-253.

SCHAEDEL, R. P., 1970 - Murales Mochicas en Pañamarca. In: 100 Años de Arqueología (R. Ravines, comp.): 309-320; Lima: Instituto de Estudios Peruanos y Petróleos del Perú.

SHIMADA, I., 1989 - Batán Grande and Cosmological Unity in the Prehistoric Central Andes. Andean Archaeology, Monograph XXVII: 163-187; Los Angeles: Institute of Archaeology, University of California. 
UBBELOHDE-DÖERING, H., 1957 - Eingeritzte Zeichen auf Tempelwänden Nord-Perus. Proceedings of the XXXII International Congress of Americanists: 405-414; Copenhagen.

UCEDA, S. \& MUJICA, E. (editores), 1994 - Moche: Propuestas y perspectivas. Actas del Primer Coloquio sobre la Cultura Moche 1993, 549p.; Lima: Universidad nacional de Trujillo-IFEA-FOMCIENCIAS. Travaux de l'Institut Francais d'Études Andines, 79. 